\title{
Parity of Sets of Mutually Orthogonal Latin Squares*
}

\author{
Nevena Francetić ${ }^{\dagger}$ Sarada Herke ${ }^{\dagger \ddagger}$ Ian M. Wanless ${ }^{\dagger}$
}

\begin{abstract}
Every Latin square has three attributes that can be even or odd, but any two of these attributes determines the third. Hence the parity of a Latin square has an information content of 2 bits. We extend the definition of parity from Latin squares to sets of mutually orthogonal Latin squares (MOLS) and the corresponding orthogonal arrays (OA). Suppose the parity of an $\operatorname{OA}(k, n)$ has an information content of $\mathcal{B}(k, n)$ bits. We show that $\mathcal{B}(k, n) \leqslant\left(\begin{array}{l}k \\ 2\end{array}\right)-1$. For the case corresponding to projective planes we prove a tighter bound, namely $\mathcal{B}(n+1, n) \leqslant\left(\begin{array}{l}n \\ 2\end{array}\right)$ when $n$ is odd and $\mathcal{B}(n+1, n) \leqslant\left(\begin{array}{l}n \\ 2\end{array}\right)-1$ when $n$ is even. Using the existence of MOLS with subMOLS, we prove that if $\mathcal{B}(k, n)=\left(\begin{array}{l}k \\ 2\end{array}\right)-1$ then $\mathcal{B}(k, N)=\left(\begin{array}{l}k \\ 2\end{array}\right)-1$ for all sufficiently large $N$.

Let the ensemble of an $\mathrm{OA}$ be the set of Latin squares derived by interpreting any three columns of the $\mathrm{OA}$ as a Latin square. We demonstrate many restrictions on the number of Latin squares of each parity that the ensemble of an $\mathrm{OA}(k, n)$ can contain. These restrictions depend on $n(\bmod 4)$ and give some insight as to why it is harder to build projective planes of order $n \equiv 2(\bmod 4)$ than for $n \not \equiv 2(\bmod 4)$. For example, we prove that when $n \equiv 2(\bmod 4)$ it is impossible to build an $\mathrm{OA}(n+1, n)$ for which all Latin squares in the ensemble are isotopic (equivalent to each other up to permutation of the rows, columns and symbols).
\end{abstract}

Keywords: parity, Latin square, MOLS, subMOLS, orthogonal array, projective plane.

\section{Introduction}

The aim of this paper is to develop a notion of parity for the orthogonal arrays that define sets of mutually orthogonal Latin squares (MOLS). The important notion of parity for permutations is widely known. A Latin square of order $n$ is an $n \times n$ square with entries in an $n$-set $\Lambda$, called the alphabet, having the property that every element of $\Lambda$ occurs exactly once in each row and each column of the square. Latin squares are 2-dimensional analogues of permutations and they too have a notion of parity. This parity plays a pivotal role in a famous conjecture of Alon and Tarsi (see e.g. [1, 21] and the references therein) and has also proved crucial in a variety of other quite distinct investigations. In [7], parity was found to explain observed limitations on which Latin squares could be embedded together in topological surfaces. In [22] and [14], parity explains large components that arise in graphs made by local switchings in Latin squares or in 1-factorisations of the complete graph, respectively. Parity considerations can also assist in diagnosing symmetries of Latin squares [16].

It is clear that parity of single Latin squares is a useful concept. It is therefore natural to try to extend this concept to sets of MOLS. A set of MOLS is a set of Latin squares such that when any two of the squares are superimposed, every ordered pair of symbols occurs exactly

${ }^{*}$ Research supported by ARC grant DP150100506

${ }^{\dagger}$ School of Mathematical Sciences, Monash University, Victoria 3800, Australia

${ }^{\ddagger}$ Current address: School of Mathematics and Physics, The University of Queensland, QLD 4072, Australia 
once. For any list $M=\left[M_{1}, \ldots, M_{k-2}\right]$ of MOLS on an alphabet $\Lambda$ we define an $n^{2} \times k$ matrix, denoted $\mathscr{A}(M)$, by taking one row $\left[r, c, M_{1}[r, c], \ldots, M_{k-2}[r, c]\right]$ for each pair $(r, c) \in \Lambda^{2}$. (For the sake of definiteness, we insist that these rows are ordered lexicographically. Also, if $M$ is given as a set rather than a list, then we impose lexicographic order on $M$ in order to create $\mathscr{A}(M)$.) Now, $\mathscr{A}(M)$ is an orthogonal array $\mathrm{OA}(k, n)$ (of strength 2 with $n$ levels and index 1) because it has the defining property that every pair of columns contains every ordered pair of elements of $\Lambda$ exactly once. Conversely, if $A$ is an $\mathrm{OA}(k, n)$ we define $\mathscr{M}(A)$ to be the set of $k-2$ MOLS formed by taking the entry in row $r$, column $c$ of the $i$-th Latin square to be the entry in column $i+2$ of the row of $A$ that begins $[r, c, \ldots]$. In this sense, an $\mathrm{OA}(k, n)$ is equivalent to a set of $k-2$ MOLS of order $n$ (see e.g. [6, III.3] for more details and background). Throughout this paper, we assume that $k$ and $n$ are integers and that $n \geqslant 2$ and $3 \leqslant k \leqslant n+1$.

Let $\mathcal{S}_{\Lambda}$ denote the permutations of $\Lambda$. Two orthogonal arrays on alphabet $\Lambda$ are isotopic if one can be obtained from the other by some sequence of operations of the following type: choose a $\gamma \in \mathcal{S}_{\Lambda}$ and a column $c$ and apply $\gamma$ to every entry in column $c$. We say two orthogonal arrays are conjugate if one can be obtained from the other by permuting the columns. We say two Latin squares are isotopic (respectively, conjugate) if their orthogonal arrays are isotopic (respectively, conjugate). We say two orthogonal arrays are isomorphic if, up to possible reordering of the rows of the arrays, one is isotopic to a conjugate of the other.

A finite projective plane of order $n$ (see e.g. [6] for the definition) can be used to define an $\mathrm{OA}(n+1, n)$ and vice versa. However, there are some subtleties to this relationship. Let $\mathcal{L}$ be a line of a finite projective plane of order $n$. We can make an $\mathrm{OA}(n+1, n)$, using $\mathcal{L}$ as the "line at infinity", as follows. First, we number the points on $\mathcal{L}$ as $p_{1}, \ldots, p_{n+1}$. Next, for $1 \leqslant i \leqslant n+1$, we number the lines (other than $\mathcal{L}$ ) through $p_{i}$, calling them $\ell_{i 1}, \ldots, \ell_{i n}$. Finally, for each point $q$ not on $\mathcal{L}$ we add a row to $A$ which has entry $i$ in column $j$ if the line through $q$ and $p_{j}$ is $\ell_{j i}$. The choices for the numbering of lines and points do not change the isomorphism class of the resulting orthogonal array. However, different choices for $\mathcal{L}$ may produce non-isomorphic orthogonal arrays. Hence, each projective plane of order $n$ can potentially produce representatives from up to $n^{2}+n+1$ isomorphism classes of $\mathrm{OA}(n+1, n)$. The reverse relationship is simpler. Every $\mathrm{OA}(n+1, n)$ can be derived in the above way from a unique projective plane.

Given a Latin square $L=\left(l_{i j}\right)$ of order $n$, we can identify 3 parities $\pi_{r}(L), \pi_{c}(L)$ and $\pi_{s}(L)$ as follows. We assume that the symbols $\Lambda$ index the rows and columns of $L$ (in this paper $\Lambda$ will either be $\{1, \ldots, n\}$ or $\left.\mathbb{Z}_{n}\right)$. Let $\pi: \mathcal{S}_{\Lambda} \rightarrow \mathbb{Z}_{2}$ denote the usual parity homomorphism with kernel the alternating group. For all $i \in \Lambda$ we can define a permutation of $\Lambda$ by $j \mapsto l_{i j}$. Applying $\pi$ to these permutations and taking the sum, mod 2, we obtain the row-parity $\pi_{r}(L)$. The column-parity $\pi_{c}(L)$ is defined similarly, using each permutation $i \mapsto l_{i j}$ formed by fixing some $j \in \Lambda$. The symbol-parity $\pi_{s}(L)$ is the sum of the parities of the permutations formed by fixing an $\ell \in \Lambda$ and mapping $i \mapsto j$ whenever $l_{i j}=\ell$. These three parities are related by

$$
\pi_{r}+\pi_{c}+\pi_{s} \equiv\left(\begin{array}{l}
n \\
2
\end{array}\right) \quad(\bmod 2)
$$

This relation has been rediscovered numerous times. Different proofs have been published in [7, 10, 13, 22, 24] and we are also aware of other researchers finding their own proof but not publishing it. We will prove a generalisation of (1.1) in Lemma 2.6, providing a new proof of the original result in the process.

By (1.1), a Latin square has one of the following parity types:

$$
\begin{array}{ll}
\pi_{r} \pi_{c} \pi_{s} \in\{000,011,101,110\} & \text { if } n \equiv 0,1(\bmod 4), \\
\pi_{r} \pi_{c} \pi_{s} \in\{111,100,010,001\} & \text { if } n \equiv 2,3(\bmod 4) .
\end{array}
$$

It is known [4] that the proportion of Latin squares which have each of the four parity types that are possible for order $n$ is $\frac{1}{4}+o(1)$ as $n \rightarrow \infty$ (for a related result, see [2]). An equiparity Latin 
square has $\pi_{r} \pi_{c} \pi_{s} \in\{000,111\}$. Loosely speaking, "nice" Latin squares have a greater than $1 / 4$ chance of being equiparity. For example, all Cayley tables of finite groups are necessarily equiparity, because they are isotopic to all of their conjugates [15, Thm 4.2.2].

In 92 , we will define two notions of parity associated with an orthogonal array. The first one we refer to as $\tau$-parity; it is a direct generalisation of the definition of parities $\pi_{r}, \pi_{c}, \pi_{s}$ for a Latin square. The second one we call $\sigma$-parity. It was introduced by Glynn and Byatt [11] (see also Glynn [10]) for $n$ even, but we define it for all $n$. We establish the precise relationship between these two seemingly different definitions of parity of an OA. For the remainder of the paper we look into properties of $\tau$-parity and $\sigma$-parity and insights that each can offer.

In $\$ 3$ we introduce graphs that are useful tools for studying both notions of parity. In 4 we consider the question of how many different $\tau$-parities an $\mathrm{OA}(k, n)$ can have. We view this question in information theory terms, by measuring the number of independent bits of information that there are in the $\tau$-parity. For example, the $\tau$-parity of an $\operatorname{OA}(3, n)$ has an information content of 2 bits given that any two of $\pi_{r}, \pi_{c}, \pi_{s}$ determine the third, by (1.1). We prove a bound on the information content of the $\tau$-parity of an $\mathrm{OA}(k, n)$, and prove that this bound is achieved for all large $n$ when $k \leqslant 5$.

In $\$ 5$ we consider the important special case of the orthogonal arrays derived from projective planes as described above. The theory developed in earlier sections is applied to this case. Stronger conclusions can be drawn than in the general case. For example, we show that the information content in the $\tau$-parity is lower than the general bound from $\$ 4$.

Let $A$ be an $\mathrm{OA}(k, n)$. The ensemble of $A$ is the set of Latin squares $\mathscr{M}(B)$, where $B$ ranges across all $\left(\begin{array}{l}k \\ 3\end{array}\right)$ choices of $\mathrm{OA}(3, n)$ formed by 3 columns of $A$ (the columns should occur in the same order in $B$ as they do in $A$ ). In $\$ 6$ we investigate the parities of the Latin squares in the ensemble of $A$. We find significant restrictions on the number of equiparity Latin squares, particularly in the case when $k=n+1$. Among other things, these allow us to deduce that for $n \equiv 2(\bmod 4)$ there is no $\mathrm{OA}(n+1, n)$ for which all Latin squares in the ensemble are isotopic to each other. This is in contrast with the $n \not \equiv 2(\bmod 4)$ case, where the Desarguesian projective planes provide examples of $\mathrm{OA}(n+1, n)$ for which all Latin squares in the ensemble are isotopic to the Cayley table of the elementary abelian group of order $n$.

\section{Two notions of parity}

Throughout the paper, we use the discrete interval notation $[a, b]=\{a, a+1, \ldots, b\}$ for $a, b \in \mathbb{Z}$. In this section we define two notions of parity for orthogonal arrays and demonstrate the relationship between them. The two notions of parity are called $\tau$-parity and $\sigma$-parity. Each $\tau_{i j}^{c}$ of the $\tau$-parity and each $\pi\left(\sigma_{i j}\right)$ of the $\sigma$-parity is an element of $\mathbb{Z}_{2}$ and hence, throughout this paper, every equation involving $\tau$-parities or $\sigma$-parities is assumed to be calculated in $\mathbb{Z}_{2}$. Also, because it makes the definition of $\sigma$-parity more natural, we will choose to always index the rows of an OA by $\Lambda^{2}$, where $\Lambda$ is the alphabet.

\section{$2.1 \quad \tau$-parity}

Definition 2.1 ( $\tau$-parity). Let $A=\left(a_{r \ell}\right)$ be an $\mathrm{OA}(k, n)$ on an alphabet $\Lambda$, where $r \in \Lambda^{2}$ and $\ell \in[1, k]$. For each ordered triple $(c, i, j)$ of distinct numbers in $[1, k]$ and for each $s \in \Lambda$, define $\rho_{s}(c, i, j)$ to be the permutation of $\Lambda$ mapping $a_{r i} \mapsto a_{r j}$ whenever $a_{r c}=s$. Define $\tau_{i j}^{c}=\tau_{i j}^{c}(A)$ as the sum of the parities of these $n$ permutations; that is, $\tau_{i j}^{c}=\sum_{s \in \Lambda} \pi\left(\rho_{s}(c, i, j)\right)$. We refer to the vector of parities $\tau_{i j}^{c}$ indexed by the $k(k-1)(k-2)$ triples $(c, i, j)$ as the $\tau$-parity of $A$.

Note that $\left\{a_{r i}: a_{r c}=s\right\}=\Lambda=\left\{a_{r j}: a_{r c}=s\right\}$ by the definition of an orthogonal array. Hence, $\left\{\rho_{s}(c, i, j): s \in \Lambda\right\}$ is indeed a set of $n$ well-defined permutations. 
The $\tau$-parity of an orthogonal array $A$ naturally extends the notion of the parity of a single Latin square to a set of MOLS. If $M_{1}, \ldots, M_{k-2}$ are the MOLS in $\mathscr{M}(A)$ then for the Latin square $M_{i-2}$ we have $\pi_{r}=\tau_{2 i}^{1}, \pi_{c}=\tau_{1 i}^{2}$, and $\pi_{s}=\tau_{12}^{i}$. For distinct $c, i, j, \ell \in[1, k]$ we have

$$
\begin{aligned}
& \tau_{i j}^{c}=\tau_{j i}^{c}, \\
& \tau_{i j}^{c}=\tau_{i \ell}^{c}+\tau_{\ell j}^{c} .
\end{aligned}
$$

The first equation follows from the fact that a permutation and its inverse have the same parity. The second equation follows from composition of permutations.

Next we consider $\tau$-parities of isomorphic orthogonal arrays. Permuting the rows of an orthogonal array $A$ has no effect on $\mathscr{M}(A)$, and permuting the symbols within a column results in an isotopic set of MOLS. Permuting the columns yields a conjugate set of MOLS. We investigate the effect of these basic operations on the $\tau$-parity.

Lemma 2.2. Let $A_{1}$ and $A_{2}$ be two orthogonal arrays, $\mathrm{OA}(k, n)$, on alphabet $\Lambda$. Index the columns of $A_{1}$ and $A_{2}$ by $[1, k]$. Let $c, i, j \in[1, k]$ be distinct integers.

(i) If $A_{2}$ is obtained by permuting the rows of $A_{1}$, then $\tau_{i j}^{c}\left(A_{2}\right)=\tau_{i j}^{c}\left(A_{1}\right)$.

(ii) If $A_{2}$ is obtained by permuting the columns of $A_{1}$ by $\gamma$, then $\tau_{\gamma(i) \gamma(j)}^{\gamma(c)}\left(A_{2}\right)=\tau_{i j}^{c}\left(A_{1}\right)$.

(iii) Let $\gamma \in \mathcal{S}_{\Lambda}$ and $c \in[1, k]$. If $A_{2}$ is obtained by applying $\gamma$ to every entry in column $c$ of $A_{1}$, then

$$
\begin{aligned}
\tau_{i j}^{c}\left(A_{2}\right) & =\tau_{i j}^{c}\left(A_{1}\right), \\
\tau_{j c}^{i}\left(A_{2}\right) & =\tau_{j c}^{i}\left(A_{1}\right)+n \cdot \pi(\gamma), \\
\tau_{i j}^{d}\left(A_{2}\right) & =\tau_{i j}^{d}\left(A_{1}\right) \text { if } d \notin\{c, i, j\} .
\end{aligned}
$$

Proof. Statements (i) and (ii) follow immediately from the definition of $\tau$-parity. To prove statement (iii), first note that $\tau_{i j}^{c}\left(A_{1}\right)$ and $\tau_{i j}^{c}\left(A_{2}\right)$ are each the sum of the parities of the same set of $n$ permutations of $\Lambda$, hence $\tau_{i j}^{c}\left(A_{2}\right)=\tau_{i j}^{c}\left(A_{1}\right)$. It is also clear that $\tau_{i j}^{d}\left(A_{2}\right)=\tau_{i j}^{d}\left(A_{1}\right)$ for $d \notin\{c, i, j\}$. Finally, in $\mathbb{Z}_{2}$ we have

$$
\tau_{j c}^{i}\left(A_{2}\right)=\sum_{s \in \Lambda} \pi\left(\gamma \rho_{s}(i, j, c)\right)=\sum_{s \in \Lambda} \pi\left(\rho_{s}(i, j, c)\right)+\sum_{s \in \Lambda} \pi(\gamma)=\tau_{j c}^{i}\left(A_{1}\right)+n \cdot \pi(\gamma)
$$

Suppose $M=\left[M_{1}, M_{2}, \ldots, M_{k}\right]$ are MOLS and that $A=\mathscr{A}(M)$ is the corresponding OA $(k+$ $2, n)$. Let $A^{\prime}$ be the $\mathrm{OA}(k+2, n)$ obtained by permuting the columns of $A$ by some permutation $\gamma$. Suppose $M^{\prime}=\mathscr{M}\left(A^{\prime}\right)=\left[M_{1}^{\prime}, M_{2}^{\prime}, \ldots, M_{k}^{\prime}\right]$. Let $\pi_{r, i}, \pi_{c, i}, \pi_{s, i}$ denote, respectively, the row, column and symbol parity of $M_{i}$ for $i \in[1, k]$, and let $\pi_{r, i}^{\prime}, \pi_{c, i}^{\prime}, \pi_{s, i}^{\prime}$ be the corresponding parities of $M_{i}^{\prime}$. It will follow from our work in $\$ 4$ that there is not enough information in $\left\{\pi_{r, i}, \pi_{c, i}, \pi_{s, i}\right.$ : $i \in[1, k]\}$ to determine $\left\{\pi_{r, i}^{\prime}, \pi_{c, i}^{\prime}, \pi_{s, i}^{\prime}: i \in[1, k]\right\}$ for all $\gamma$. However, there are some things we can say:

(i) If the first two columns are fixed points of $\gamma$ then $M^{\prime}$ is simply a reordering of the Latin squares in $M$, and the parities will be permuted accordingly.

(ii) If $\gamma$ is the transposition (12) then $M_{i}^{\prime}$ is the transpose of $M_{i}$, so $\pi_{r, i}^{\prime}=\pi_{c, i}, \pi_{c, i}^{\prime}=\pi_{r, i}$ and $\pi_{s, i}^{\prime}=\pi_{s, i}$ for $i \in[1, k]$.

(iii) If $\gamma$ is the transposition (23) then $M_{1}^{\prime}$ is a conjugate of $M_{1}$ for which $\pi_{r, 1}^{\prime}=\pi_{r, 1}, \pi_{c, 1}^{\prime}=\pi_{s, 1}$ and $\pi_{s, 1}^{\prime}=\pi_{c, 1}$. Moreover, $\pi_{r, i}^{\prime}=\pi_{r, i}+\pi_{r, 1}$ for $i \in[2, k]$, because $\tau_{2(i+2)}^{1}=\tau_{23}^{1}+\tau_{3(i+2)}^{1}$ by $(2.2)$.

Note that any permutation of the columns can be achieved by composing the permutations in (i), (ii) and (iii) above. 


\section{$2.2 \sigma$-parity}

In this subsection, we consider an alternative definition of parity of an orthogonal array. This definition was introduced by Glynn and Byatt [11] (see also Glynn [10]) for orthogonal arrays with even alphabet size, although it is also useful for odd alphabet sizes.

Definition 2.3 ( $\sigma$-parity). Let $A=\left(a_{r \ell}\right)$ be an $\mathrm{OA}(k, n)$ on alphabet $\Lambda$, where $r \in \Lambda^{2}$ and $\ell \in[1, k]$. Let $i, j \in[1, k]$ be two distinct integers. Then $\sigma_{i j}: \Lambda^{2} \rightarrow \Lambda^{2}$ is the permutation defined by $\sigma_{i j}(r)=\left(a_{r i}, a_{r j}\right)$. We sometimes write $\sigma_{i j}^{A}$ for $\sigma_{i j}$ to stress the role that $A$ plays. We refer to the vector of parities $\pi\left(\sigma_{i j}\right)$ indexed by the $k(k-1)$ pairs $(i, j)$ as the $\sigma$-parity of $A$.

In $\$ 2.3$, we establish an equivalence between $\tau$-parity and $\sigma$-parity. Here, we consider some basic properties of $\sigma$-parity. First, we observe the effect of interchanging the indices.

Lemma 2.4. Given an $\mathrm{OA}(k, n)$ and distinct integers $i, j \in[1, k]$,

$$
\pi\left(\sigma_{j i}\right)=\pi\left(\sigma_{i j}\right)+\left(\begin{array}{l}
n \\
2
\end{array}\right) .
$$

Proof. Let $A=\left(a_{r \ell}\right)$ be an $\mathrm{OA}(k, n)$ on alphabet $\Lambda$, where $r \in \Lambda^{2}$ and $\ell \in[1, k]$. Since $A$ is an OA, there is a bijection $R: \Lambda^{2} \rightarrow \Lambda^{2}$ for which $R(u, v)=r$ where $u=a_{r i}$ and $v=a_{r j}$. Then $\sigma_{j i}=\sigma_{i j} \prod_{\{u, v\}}(R(u, v), R(v, u))$, where $(R(u, v), R(v, u))$ is a transposition and the product is over all unordered pairs of distinct elements of $\Lambda$. The claim follows.

Note that (2.3) relies on an essential property of orthogonal arrays; namely, that every ordered pair of distinct symbols occurs exactly once in every pair of columns of an OA. We refer to this equation to derive further properties of both $\sigma$ - and $\tau$-parities.

Next, we consider the analogue of Lemma 2.2 for $\sigma$-parity; that is, we consider the $\sigma$-parities of isomorphic orthogonal arrays.

Lemma 2.5. Let $A_{1}$ and $A_{2}$ be two orthogonal arrays, $\mathrm{OA}(k, n)$, on an alphabet $\Lambda$. Index the columns of $A_{1}$ and $A_{2}$ by $[1, k]$. Let $i, j \in[1, k]$ be two distinct integers.

(i) If $A_{2}$ is obtained by permuting the rows of $A_{1}$ by $\gamma$, then $\pi\left(\sigma_{i j}^{A_{2}}\right)=\pi\left(\sigma_{i j}^{A_{1}}\right)+\pi(\gamma)$.

(ii) If $A_{2}$ is obtained by permuting the columns of $A_{1}$ by $\gamma$, then $\pi\left(\sigma_{\gamma(i) \gamma(j)}^{A_{2}}\right)=\pi\left(\sigma_{i j}^{A_{1}}\right)$.

(iii) Let $\gamma \in \mathcal{S}_{\Lambda}$ and $i \in[1, k]$. If $A_{2}$ is obtained by applying $\gamma$ to every entry in column $i$ of $A_{1}$, then $\pi\left(\sigma_{i j}^{A_{2}}\right)=\pi\left(\sigma_{i j}^{A_{1}}\right)+n \cdot \pi(\gamma)$, and if $i^{\prime} \neq i$ then $\pi\left(\sigma_{i^{\prime} j}^{A_{2}}\right)=\pi\left(\sigma_{i^{\prime} j}^{A_{1}}\right)$.

Proof. If $\gamma$ is a permutation of $\Lambda^{2}$ which acts on the rows of $A_{1}$, then $\sigma_{i j}^{A_{2}}=\sigma_{i j}^{A_{1}} \gamma$, which implies the first statement. Similarly, if $\gamma$ is a permutation of $[1, k]$ which acts on the columns of $A_{2}$, then $\sigma_{i j}^{A_{1}}=\sigma_{\gamma(i) \gamma(j)}^{A_{2}}$. Now assume that $\gamma$ is a permutation of $\Lambda$ that is applied to every entry in column $i$ of $A_{1}=\left(a_{r \ell}\right)$. Write $\gamma$ as a product of transpositions: $\gamma=\left(v_{1}, v_{1}^{\prime}\right)\left(v_{2}, v_{2}^{\prime}\right) \cdots\left(v_{m}, v_{m}^{\prime}\right)$ where $m \geqslant 0$ and $v_{l} \neq v_{l}^{\prime}$ for $l \in[1, m]$. Let $R$ be the bijection from the proof of Lemma 2.4. Then

$$
\sigma_{i j}^{A_{2}}=\sigma_{i j}^{A_{1}} \prod_{l \in[1, m]} \prod_{s \in \Lambda}\left(R\left(v_{l}, s\right), R\left(v_{l}^{\prime}, s\right)\right) .
$$

Therefore, $\pi\left(\sigma_{i j}^{A_{2}}\right)=\pi\left(\sigma_{i j}^{A_{1}}\right)+m n=\pi\left(\sigma_{i j}^{A_{1}}\right)+n \cdot \pi(\gamma)$ in $\mathbb{Z}_{2}$. The claim is obvious for $i^{\prime} \neq i$. 


\subsection{Equivalence between $\tau$-parity and $\sigma$-parity}

In this subsection, we show that the $\sigma$-parity of an orthogonal array determines its $\tau$-parity. Also, the converse statement is true up to complementation.

Let $A=\left(a_{r \ell}\right)$ be an $\mathrm{OA}(k, n)$ and fix distinct $c, i, j \in[1, k]$. Consider a row $r$ of $A$ and let $x=a_{r c}, y=a_{r i}$ and $z=a_{r j}$. The permutation $\sigma_{c j} \sigma_{c i}^{-1}$ maps $(x, y)$ to $(x, z)$. Thus the contribution to $\pi\left(\sigma_{c j} \sigma_{c i}^{-1}\right)$ from the rows in which the symbol $x$ occurs in column $c$ is precisely $\pi\left(\rho_{x}(c, i, j)\right)$. Summing over $x$, we find that

$$
\tau_{i j}^{c}=\pi\left(\sigma_{c j} \sigma_{c i}^{-1}\right)=\pi\left(\sigma_{c i} \sigma_{c j}\right) .
$$

This demonstrates that $\sigma$-parity determines $\tau$-parity.

Note that Lemma 2.5(i) implies that if two rows of an OA are interchanged, then every $\pi\left(\sigma_{i j}\right)$ changes value. We call this $\sigma$-complementation. However, interchanging two rows of an orthogonal array $A$ does not change $\mathscr{M}(A)$, hence one would not expect the parity to change. Also, permuting the rows of an OA does not affect $\tau$-parity (see Lemma 2.2(i)), which means there is no hope of recovering $\sigma$-parity from $\tau$-parity. However, if some kind of standardisation is imposed to choose between a $\sigma$-parity and its $\sigma$-complement, then this standardised choice may be recovered from the $\tau$-parity. We will use two forms of standardisation in this paper. The simplest is just to insist that $\pi\left(\sigma_{12}\right)=0$. Under this convention, it is easy to recover the $\sigma$-parity given the $\tau$-parity of an orthogonal array, as follows:

$$
\begin{array}{ll}
\pi\left(\sigma_{12}\right)=0, & \\
\pi\left(\sigma_{1 j}\right)=\tau_{2 j}^{1} & \text { for } j \geqslant 3, \\
\pi\left(\sigma_{2 j}\right)=\tau_{1 j}^{2}+\left(\begin{array}{l}
n \\
2
\end{array}\right) & \text { for } j \geqslant 3, \\
\pi\left(\sigma_{i j}\right)=\tau_{2 i}^{1}+\tau_{1 j}^{i}+\left(\begin{array}{l}
n \\
2
\end{array}\right) & \text { for } 3 \leqslant i<j .
\end{array}
$$

As usual, all parity equations are in $\mathbb{Z}_{2}$. The first of these four equations, (2.5), is our standardisation. By (2.4),$\tau_{2 j}^{1}=\pi\left(\sigma_{12} \sigma_{1 j}\right)=\pi\left(\sigma_{12}\right)+\pi\left(\sigma_{1 j}\right)$ when $j \geqslant 3$, giving (2.6). Note that $\tau_{2 j}^{1}+\tau_{1 j}^{i}=\pi\left(\sigma_{12} \sigma_{1 j} \sigma_{i 1} \sigma_{i j}\right)$. When $i=2$ and $j \geqslant 3$, using (2.5) and (2.6) as well as the property (2.3), we have

$$
\begin{aligned}
\tau_{2 j}^{1}+\tau_{1 j}^{2} & =\pi\left(\sigma_{1 j}\right)+\pi\left(\sigma_{21}\right)+\pi\left(\sigma_{2 j}\right) \\
& =\tau_{2 j}^{1}+\left(\begin{array}{l}
n \\
2
\end{array}\right)+\pi\left(\sigma_{2 j}\right),
\end{aligned}
$$

which gives (2.7). Similarly, if $i \geqslant 3$, we have

$$
\begin{aligned}
\tau_{2 j}^{1}+\tau_{1 j}^{i} & =\pi\left(\sigma_{1 j}\right)+\pi\left(\sigma_{i 1}\right)+\pi\left(\sigma_{i j}\right) \\
& =\tau_{2 j}^{1}+\tau_{2 i}^{1}+\left(\begin{array}{l}
n \\
2
\end{array}\right)+\pi\left(\sigma_{i j}\right),
\end{aligned}
$$

which gives (2.8) .

In several arguments towards the end of $₫ 6$ it will be convenient to use a form of standardisation other than (2.5) . However, any method which decides whether to take a $\sigma$-parity or its $\sigma$-complement will allow $\sigma$-parity to be recovered from $\tau$-parity. We can simply find the answer determined by (2.5)-(2.8) and then take the $\sigma$-complement or not, as required.

We can use the relationship between $\tau$-parity and $\sigma$-parity to generalise (1.1), and give a simple proof. 
Lemma 2.6. Suppose $c_{1}, c_{2}, \ldots, c_{l} \in[1, k]$ are distinct integers, where $l \geqslant 3$. For any $\mathrm{OA}(k, n)$ we have, in $\mathbb{Z}_{2}$,

$$
\tau_{c_{l} c_{2}}^{c_{1}}+\tau_{c_{1} c_{3}}^{c_{2}}+\cdots+\tau_{c_{i-1} c_{i+1}}^{c_{i}}+\cdots+\tau_{c_{l-1} c_{1}}^{c_{l}}=l\left(\begin{array}{l}
n \\
2
\end{array}\right)
$$

Proof. By (2.4) and (2.3),

$$
\tau_{c_{l} c_{2}}^{c_{1}}+\cdots+\tau_{c_{i-1} c_{i+1}}^{c_{i}}+\cdots+\tau_{c_{l-1} c_{1}}^{c_{l}}=\pi\left(\sigma_{c_{1} c_{l}} \sigma_{c_{l} c_{1}}\right)+\sum_{i \in[1, l-1]} \pi\left(\sigma_{c_{i} c_{i+1}} \sigma_{c_{i+1} c_{i}}\right)=l\left(\begin{array}{l}
n \\
2
\end{array}\right) .
$$

Applying Lemma 2.6 in the case where $l=3$, we find that for an $\mathrm{OA}(k, n)$ and three distinct integers $c, i, j \in[1, k]$ we have

$$
\tau_{i j}^{c}+\tau_{c j}^{i}+\tau_{c i}^{j}=\left(\begin{array}{l}
n \\
2
\end{array}\right) .
$$

Of course, this is essentially a restatement of (1.1).

\section{Graphs that model parities}

We next describe graph theoretic interpretations for both our notions of parity ( $\tau$-parity and $\sigma$-parity). Our graphs will not include loops or multiple edges, but will sometimes have directed edges. Given a graph or digraph $G$, we use $V(G)$ for the vertex set of $G$ and $E(G)$ for the set of (possibly directed) edges. For an undirected graph $G$ we use $N_{G}(v) \subseteq V(G)$ to denote the neighbourhood of $v$ in $G$, the set of vertices in $G$ which are adjacent to a given vertex $v$.

The complement of a undirected graph $G$, denoted $\bar{G}$, is the graph obtained by replacing edges of $G$ with non-edges and vice versa. By switching an undirected $G$ at $v \in V(G)$ we obtain the graph, denoted $G^{v}$, which is equal to $G$ except that the neighbourhood of $v$ in $G^{v}$ is the complement of the neighbourhood of $v$ in $G$. In other words, $N_{G^{v}}(v)=N_{\bar{G}}(v)$.

The reverse of a digraph $G$, also denoted $\bar{G}$, is the digraph obtained from $G$ by reversing the direction of every edge. By switching a directed $G$ at $v \in V(G)$, we obtain the digraph, denoted $G^{v}$, which is equal to $G$ except that the direction of each edge incident with $v$ is reversed. Note that we use the same notation $\bar{G}$ and $G^{v}$, where the meaning is determined by context, depending on whether $G$ is a undirected graph or a digraph.

For a fixed initial digraph, the digraph obtained by applying a finite sequence of switchings and reversals depends only on the parity of the number of switchings taken at each vertex, and the parity of the number of reversals taken. It does not depend on the order in which these operations are applied (see [17] for further details). It is easy to check that analogous properties hold in the context of undirected graphs as well.

\subsection{Graphs related to $\tau$-parity}

Definition 3.1. Let $A$ be an $\operatorname{OA}(k, n)$. For each $c \in[1, k]$, we define an undirected graph $G_{c}$ with $V\left(G_{c}\right)=[1, k]$, where vertex $c$ is isolated and $\{i, j\} \in E\left(G_{c}\right)$ if and only if $\tau_{i j}^{c}=1$ for $i, j \neq c$. We call the graphs $G_{1}, \ldots, G_{k}$ the $\tau$-graphs of $A$.

We find it convenient to consider empty graphs to be complete bipartite graphs (with one side of the graph having cardinality zero).

Lemma 3.2. Let $G_{1}, \ldots, G_{k}$ be the $\tau$-graphs of an $\mathrm{OA}(k, n)$. Then, for each $c \in[1, k]$, graph $G_{c}$ is the disjoint union of an isolated vertex and a complete bipartite graph on $k-1$ vertices. 
Proof. For some $c \in[1, k]$, let $G$ be $G_{c}$ with the isolated vertex $c$ removed. If $G$ has no edges, then $G$ is $K_{0, k-1}$, so assume that $G$ has at least one edge. Let $i \in V(G)$ be such that $V_{1}=N_{G}(i) \neq \emptyset$. By (2.2), there is an even number of edges between every triple of vertices in $G$, hence $V_{1}$ is an independent set. Now consider $V_{2}=V(G) \backslash V_{1}$. For every $j \in V_{2} \backslash\{i\}$ and $\ell \in V_{1}$, we know that $\{j, \ell\} \in E(G)$ because $j \notin V_{1}=N_{G}(i)$ and there must be an even number of edges induced by the vertices $\{i, j, \ell\}$. Finally, $V_{2}=N_{G}(\ell)$ for $\ell \in V_{1}$, so it is an independent set. Thus $G$ is a complete bipartite graph with partite sets $V_{1}$ and $V_{2}$.

We next state the analogue of Lemma 2.2 for $\tau$-graphs.

Lemma 3.3. Let $A_{1}$ and $A_{2}$ be $\mathrm{OA}(k, n)$ and let $G_{1}, \ldots, G_{k}$ be the $\tau$-graphs of $A_{1}$ and $H_{1}, \ldots, H_{k}$ be the $\tau$-graphs of $A_{2}$.

(i) If $A_{2}$ is obtained by permuting the rows of $A_{1}$, then $G_{c}$ is equal to $H_{c}$ for each $c \in[1, k]$.

(ii) If $A_{2}$ is obtained by permuting columns of $A_{1}$ by $\gamma$, then $\gamma$ is an isomorphism which maps $G_{c}$ to $H_{\gamma(c)}$ for all $c \in[1, k]$.

(iii) Let $\gamma \in \mathcal{S}_{\Lambda}$ and $c \in[1, k]$ and suppose $A_{2}$ is obtained by applying $\gamma$ to every entry in column $c$ of $A_{1}$. If $n$ is even or $\pi(\gamma)=0$, then $H_{i}=G_{i}$ for all $i \in[1, k]$. If $n$ is odd and $\pi(\gamma)=1$ then $H_{c}=G_{c}$ and, for $i \in[1, k] \backslash\{c\}$, we obtain $H_{i}$ by switching $G_{i} \backslash\{i\}$ at vertex $c$, with vertex $i$ remaining isolated.

We remark that the only operation that may produce isomorphic OAs with non-isomorphic $\tau$-graphs is an odd permutation of the symbols in a column when the alphabet size is odd. This results in switchings at the vertex that corresponds to the column being permuted.

The $\tau$-graphs have the same vertex set, and by Lemma 3.2, they are a disjoint union of an isolated vertex and a complete bipartite graph. Next we study what happens if we superimpose them, modulo 2. Define the stack corresponding to an $\mathrm{OA}(k, n)$ to be the undirected graph with vertices $[1, k]$ and an edge $\{i, j\}$ if and only if $\sum_{c} \tau_{i j}^{c} \equiv 1(\bmod 2)$ (in other words, the edge $\{i, j\}$ is present in an odd number of the $\tau$-graphs for the OA). The following lemma is an interesting observation about the stack corresponding to an $\mathrm{OA}(k, n)$.

Theorem 3.4. Let $G$ be the stack for an $\mathrm{OA}(k, n)$. Then

(i) $G$ is a complete bipartite graph if $n \equiv 0,1(\bmod 4)$, and

(ii) $G$ is a vertex disjoint union of at most two complete graphs if $n \equiv 2,3(\bmod 4)$.

Proof. We consider the number of edges between any three distinct vertices $i, j, \ell \in[1, k]$. Working in $\mathbb{Z}_{2}$, we have,

$$
\begin{aligned}
\sum_{c \neq i, j} \tau_{i j}^{c}+\sum_{c \neq j, \ell} \tau_{j \ell}^{c}+\sum_{c \neq \ell, i} \tau_{\ell i}^{c} & =\pi\left(\prod_{c \neq i, j} \sigma_{c i} \sigma_{c j} \prod_{c \neq j, \ell} \sigma_{c j} \sigma_{c \ell} \prod_{c \neq \ell, i} \sigma_{c \ell} \sigma_{c i}\right) & & \text { by (2.4) } \\
& =\pi\left(\sigma_{\ell i} \sigma_{\ell j} \sigma_{i j} \sigma_{i \ell} \sigma_{j \ell} \sigma_{j i}\right) & & \\
& =3\left(\begin{array}{l}
n \\
2
\end{array}\right) . & & \text { by (2.3) }
\end{aligned}
$$

Therefore, when $n \equiv 0,1(\bmod 4)$, there is an even number of edges between any three distinct vertices in $G$. Analogous to the proof of Lemma 3.2, this implies that $G$ must be a complete bipartite graph.

If $n \equiv 2,3(\bmod 4)$, there is an odd number of edges between any three distinct vertices in $G$. In particular, there are no induced paths of two edges. Since there cannot be two vertices at distance 2 from each other, it follows that each component of $G$ is a complete graph. There are at most two components, since otherwise there would be three vertices inducing a graph with no edges, but any three vertices must induce an odd number of edges. 
In Corollary 5.2 we give a stronger restriction on the stack corresponding to an $\mathrm{OA}(n+1, n)$.

\subsection{Graphs related to $\sigma$-parity}

Definition 3.5. Let $A$ be an $\mathrm{OA}(k, n)$. The $\sigma$-matrix of $A$ is the $k \times k$ matrix $M=\left(m_{i j}\right)$ where

$$
m_{i j}= \begin{cases}1 & \text { if } i \neq j \text { and } \pi\left(\sigma_{i j}\right)=1 \\ 0 & \text { otherwise. }\end{cases}
$$

The $\sigma$-graph of $A$, denoted by $\mathscr{G}(A)$, is a graph or digraph on $k$ vertices with adjacency matrix $M$. If $M$ is symmetric, then we interpret $\mathscr{G}(A)$ to be an undirected graph. Otherwise, we interpret $\mathscr{G}(A)$ to be a digraph.

Lemma 3.6. Let $A$ be an $\mathrm{OA}(k, n)$. If $n \equiv 0,1(\bmod 4)$, then the $\sigma$-matrix is symmetric and the $\sigma$-graph is an undirected graph. If $n \equiv 2,3(\bmod 4)$, then the $\sigma$-graph is a tournament.

Proof. By (2.3), we have $\pi\left(\sigma_{j i}\right)=\pi\left(\sigma_{i j}\right)+\left(\begin{array}{l}n \\ 2\end{array}\right)$ for distinct $i, j \in[1, k]$. If $n \equiv 0,1(\bmod 4)$, then $\sigma_{i j}$ and $\sigma_{j i}$ have the same parity, so the $\sigma$-matrix of $A$ is symmetric. If $n \equiv 2,3(\bmod 4)$, then $\sigma_{i j}$ and $\sigma_{j i}$ have opposite parity, so exactly one of the directed edges $(i, j)$ and $(j, i)$ is in $\mathscr{G}(A)$. Hence $\mathscr{G}(A)$ is a tournament.

We next state the analogue of Lemma 2.5 for $\sigma$-graphs. Glynn and Byatt [11, Lem. 2.4] showed that $\sigma$-graphs of orthogonal arrays $\mathrm{OA}(k, n)$ for $n$ even are invariant (up to complementation) for isomorphic orthogonal arrays. Here we consider $n$ odd as well. The proof is essentially the same as for the even case, so we omit it.

Lemma 3.7. Let $A_{1}$ and $A_{2}$ be $\mathrm{OA}(k, n)$ and let $\mathscr{G}_{1}=\mathscr{G}\left(A_{1}\right)$ and $\mathscr{G}_{2}=\mathscr{G}\left(A_{2}\right)$.

(i) If $A_{2}$ is obtained by permuting the rows of $A_{1}$ by $\gamma$, then $\mathscr{G}_{2}$ is either $\mathscr{G}_{1}$ if $\gamma$ is an even permutation, or $\overline{\mathscr{G}_{1}}$ if $\gamma$ is an odd permutation.

(ii) If $A_{2}$ is obtained by permuting the columns of $A_{1}$ by $\gamma$, then $\gamma$ is a graph isomorphism which maps $\mathscr{G}_{1}$ to $\mathscr{G}_{2}$.

(iii) Let $\gamma \in \mathcal{S}_{\Lambda}$ and $i \in[1, k]$ and suppose $A_{2}$ is obtained by applying $\gamma$ to every entry in column $i$ of $A_{1}$. Then $\mathscr{G}_{2}=\mathscr{G}_{1}$ if $\pi(\gamma)=0$ or $n$ is even, and $\mathscr{G}_{2}=\mathscr{G}_{1}^{i}$ if $\pi(\gamma)=1$ and $n$ is odd.

We see in Lemma 3.7 that taking a conjugate of a set of MOLS only affects the order of columns of the orthogonal arrays, and hence yields an isomorphic $\sigma$-graph. On the other hand, the $\sigma$-graphs of isotopic sets of MOLS are not necessarily isomorphic if $n$ is odd, because isotopisms can cause switchings at some vertices.

\section{How many parities are there?}

If $A$ is an $\mathrm{OA}(k, n)$ then there is a corresponding $\tau$-parity $\tau(A)$ of dimension $k(k-1)(k-2)$ which stores the parities $\tau_{i j}^{c}$, indexed by the triples $(c, i, j)$. In this section we are interested in the number of different $\tau$-parities that are possible. One way to measure this is to consider $\mathcal{B}(k, n)$, which we define as $\log _{2}$ of the number of different $\tau$-parities achieved by orthogonal arrays $\mathrm{OA}(k, n)$. We have already determined a number of relationships between components of the $\tau$-parity, including (2.1), (2.2) and (2.9)). Studying $\mathcal{B}(k, n)$ is one way to determine whether there are other relationships waiting to be discovered. We will be able to resolve this question in some cases, but it will remain open in general. Another way to view $\mathcal{B}(k, n)$ is that it is the information content (in number of bits) of $\tau(A)$ for an $\mathrm{OA}(k, n)$. 
For a given value of $(k, n)$, we call any $\mathbb{Z}_{2}$ vector satisfying (2.1), (2.2) and (2.9) a plausible $\tau$-parity. We call it an actual $\tau$-parity if there is an $\mathrm{OA}(k, n)$ that achieves it. The set of plausible $\tau$-parities depends only on the value of $n(\bmod 4)$ and on $k$.

\subsection{Switching classes}

Once we have an actual $\tau$-parity, there will be many other actual $\tau$-parities that we can establish by taking isomorphic orthogonal arrays. For a plausible $\tau$-parity $p$, the switching class of $p$ is the set of all plausible $\tau$-parities obtained from $p$ by some sequence of the following operations. These operations are analogues of (ii) and (iii) from Lemma 2.2. and will map any actual $\tau$-parity to another actual $\tau$-parity.

Permuting Operation: For a permutation $\gamma \in \mathcal{S}_{k}$, a new $\tau$-parity is obtained by permuting by $\gamma$ as follows: we move each $\tau_{i j}^{c}$ from the coordinate indexed by $(c, i, j)$ to the coordinate indexed by $(\gamma(c), \gamma(i), \gamma(j))$.

Swapping Operation: If $n$ is odd then a new $\tau$-parity is obtained by swapping at a subset $C \subseteq[1, k]$, which means for each $\tau_{i j}^{c}$, we change its value if and only if $|\{i, j\} \cap C|=1$.

We stress that the swapping operation is only available if $n$ is odd.

Theorem 4.1. Let $k \geqslant 3$ and $n \geqslant 2$. Then each switching class for parameters $(k, n)$ has size which divides $k$ ! if $n$ is even and $k ! 2^{k-1}$ if $n$ is odd.

Proof. First, suppose that $n$ is even. The group $\mathcal{S}_{k}$ acting on the labels $c, i, j$, induces an action on the $\tau$-parities. The switching classes are the orbits of this action. Hence the result follows.

The situation for odd $n$ is similar, except that we have the option to swap at any subset $C \subseteq[1, k]$. However, swapping at $C$ has the same effect as swapping at the complement of $C$. We can remove this duplication by marking one index in $[1, k]$, and never swapping at the marked index (the mark gets moved when we permute indices). In this way we find an action of the group $\mathcal{S}_{2}^{k-1} \rtimes \mathcal{S}_{k}$ on $\tau$-parities. This group has order $k ! 2^{k-1}$ and its orbits are the switching classes.

We remark that $\mathcal{S}_{2}^{k-1} \rtimes \mathcal{S}_{k}$ is the automorphism group of the folded $k$-cube (at least when $k>4$ ), see [3, pp. 264-265].

For $k \in[3,8]$ and for each possible value of $n(\bmod 4)$, we calculated the number of switching classes and the size of each switching class for every plausible $\tau$-parity. The results are given in Table 1. Instances that achieve the bounds in Theorem 4.1 are shown in bold. Note that for odd $n$ the bounds are not achieved until $k=8$.

Each plausible $\tau$-parity corresponds to a $\sigma$-graph up to complementation, and vice versa. For even $n$, a switching class is defined by the permuting operation, which simply applies an isomorphism to the $\sigma$-graph. It follows that the number of switching classes for $(k, n)$ when $n \equiv 0(\bmod 4)$ is the number of complementary pairs of graphs on $k$ nodes (sequence A007869 in [19]). Similarly, the number of switching classes for $(k, n)$ when $n \equiv 2(\bmod 4)$ is the number of complementary pairs of tournaments on $k$ nodes (sequence A059735 in [19]).

Up to isotopism there are 19 complete sets of MOLS of order 9, falling into 7 isomorphism classes of $\mathrm{OA}(10,9)$. For each complete set $M$ of MOLS, we constructed $\mathscr{A}(M)$, the corresponding $\mathrm{OA}(10,9)$. We then determined its $\tau$-parity, and the switching class it belongs to. The results are shown in Table 2. Sets of MOLS are numbered using the numbering in [6]. Set number 1 corresponds to the Desarguesian plane, sets 2 to 6 correspond to the Hall plane, sets 7 to 11 correspond to the dual Hall plane, and sets 12 to 19 correspond to the Hughes plane. It turns out that only 4 different switching classes are represented by the 19 complete sets. These 4 switching classes do not correspond to the 4 projective planes. In fact one of the switching 


\begin{tabular}{|c|c|c|c|c|}
\hline \multirow[t]{2}{*}{$k$} & \multicolumn{2}{|r|}{$n \equiv 0(\bmod 4)$} & \multicolumn{2}{|r|}{$n \equiv 2(\bmod 4)$} \\
\hline & $\#$ & sizes & $\#$ & sizes \\
\hline 3 & 2 & 1,3 & 2 & 1,3 \\
\hline 4 & 6 & $1,3,4,6,12$ & 3 & 8,12 \\
\hline 5 & 18 & $1,5,6,10,15,20,30,60$ & 10 & $12,20,40,60,120$ \\
\hline 6 & 78 & $1,6,10,15,20,30,45,60,72,90,120,180,360,720$ & 34 & $40,120,144,240,360, \mathbf{7 2 0}$ \\
\hline 7 & 522 & $\begin{array}{l}1,7,21,35,42,70,105,140,210,252,315,360,420 \\
504,630,840,1260,2520, \mathbf{5 0 4 0}\end{array}$ & 272 & $\begin{array}{l}120,280,360,504,560,840 \\
1008,1680,2520, \mathbf{5 0 4 0}\end{array}$ \\
\hline 8 & 6178 & $\begin{array}{l}1,8,28,35,56,70,105,168,210,280,315,336, \\
420,560,630,672,840,1120,1260,1680,2016,2520, \\
2880,3360,4032,5040,6720,10080,20160, \mathbf{4 0 3 2 0}\end{array}$ & 3528 & $\begin{array}{l}1920,2240,2688,4480 \\
5760,6720,8064,13440 \\
20160, \mathbf{4 0 3 2 0}\end{array}$ \\
\hline
\end{tabular}

\begin{tabular}{|c|c|c|c|c|}
\hline \multirow[t]{2}{*}{$k$} & \multirow{2}{*}{\multicolumn{2}{|c|}{$n \equiv 1(\bmod 4)$}} & \multicolumn{2}{|r|}{$n \equiv 3(\bmod 4)$} \\
\hline & & & $\#$ & sizes \\
\hline 3 & 1 & 4 & 1 & 4 \\
\hline 4 & 2 & 8,24 & 2 & 8,24 \\
\hline 5 & 4 & $16,96,160,240$ & 2 & 192,320 \\
\hline 6 & 10 & $32,192,320,480,1440,1920,2880,5760$ & 6 & $640,1920,2304,3840,5760$ \\
\hline 7 & 27 & $\begin{array}{l}64,1344,2240,4480,6720,13440,16128,20160, \\
23040,26880,40320,53760,80640,161280\end{array}$ & 12 & $\begin{array}{l}7680,17920,23040 \\
32256,53760,161280\end{array}$ \\
\hline 8 & 131 & $\begin{array}{l}128,3584,4480,7168,13440,21504,26880,35840, \\
40320,53760,71680,86016,107520,161280,215040, \\
258048,322560,368640,430080,645120,860160, \\
1290240,2580480, \mathbf{5 1 6 0 9 6 0}\end{array}$ & 69 & $\begin{array}{l}15360,143360,172032,215040, \\
286720,322560,368640,430080, \\
516096,645120,860160,1290240, \\
1720320,2580480, \mathbf{5 1 6 0 9 6 0}\end{array}$ \\
\hline
\end{tabular}

Table 1: Number and sizes of switching classes.

classes contain OAs from three different projective planes, and another contains OAs from two different projective planes, as indicated in the last column of Table 2. Clearly, not all OAs from a given plane end up in the same switching class.

\begin{tabular}{|c|c|l|c|}
\hline Switching Class & Size of class & Sets of 8 MOLS(9) in the class & \# Projective planes \\
\hline Class 1 & 1290240 & 1 & 1 \\
Class 2 & 483840 & $2,3,10,11$ & 2 \\
Class 3 & 512 & $4,5,6,7,8,9,17,18,19$ & 3 \\
Class 4 & 1075200 & $12,13,14,15,16$ & 1 \\
\hline
\end{tabular}

Table 2: Switching classes of complete sets of MOLS(9)

Table 2 also shows the size of each switching class and here it is slightly surprising that the most symmetric plane (the Desarguesian one) produces OAs in the largest switching class. In fact the smallest switching class contains the zero vector. Each of the non-Desarguesian planes have OAs which achieve this zero vector, but the Desarguesian plane does not.

\subsection{Achieving all plausible $\tau$-parities}

We next consider the question of how many plausible $\tau$-parities there are. After that we will consider how many of them are actual $\tau$-parities.

Theorem 4.2. There are $2^{\left(\begin{array}{c}k \\ 2\end{array}\right)-1}$ plausible $\tau$-parities for an $\mathrm{OA}(k, n)$, because each standardised $\sigma$-parity corresponds to a different plausible $\tau$-parity. 
Proof. We choose the standardisation (2.5). By $\$ 2.3$ we know that each standardised $\sigma$-parity determines a different $\tau$-parity, so we consider the number of options for the $\sigma$-matrix. Our choice for $\pi\left(\sigma_{12}\right)$ is fixed but we can freely pick $\pi\left(\sigma_{i j}\right)$ for all other $1 \leqslant i<j \leqslant k$. This gives us $2^{\left(\begin{array}{c}k \\ 2\end{array}\right)-1}$ options, but we have to check that all of them produce plausible $\tau$-parities. Suppose we have settled on values for $\pi\left(\sigma_{i j}\right)$ for $1 \leqslant i<j \leqslant k$. We then determine $\pi\left(\sigma_{i j}\right)$ for $1 \leqslant j<i \leqslant k$ from (2.3), and $\tau_{i j}^{c}$ for all distinct $c, i, j \in[1, n+1]$ from (2.4).

It is straightforward to check (2.1) and (2.2) hold. Also, in $\mathbb{Z}_{2}$,

$$
\begin{aligned}
\tau_{i j}^{c}+\tau_{c j}^{i}+\tau_{c i}^{j} & =\pi\left(\sigma_{c i}\right)+\pi\left(\sigma_{c j}\right)+\pi\left(\sigma_{i c}\right)+\pi\left(\sigma_{i j}\right)+\pi\left(\sigma_{j c}\right)+\pi\left(\sigma_{j i}\right) & & \text { by (2.4) } \\
& =3\left(\begin{array}{l}
n \\
2
\end{array}\right), & & \text { by (2.3) }
\end{aligned}
$$

and hence (2.9) is satisfied.

Corollary 4.3. We have $\mathcal{B}(k, n) \leqslant\left(\begin{array}{l}k \\ 2\end{array}\right)-1$ for $k \geqslant 3$ and arbitrary $n$.

In Corollary 5.6 we find a better upper bound on $\mathcal{B}(k, n)$ in the case when $k=n+1$.

We now know there are $2^{\left(\begin{array}{c}k \\ 2\end{array}\right)-1}$ plausible $\tau$-parities. How many of these are actual $\tau$-parities? In Table 3, we list some values of $(k, n)$ for which all plausible $\tau$-parities are actual. Examples that justify each claim made in the table can be downloaded from [23]. This table is exhaustive for $n \leqslant 9$, where a complete catalogue of MOLS is known [9, 23]. For $n \geqslant 10$ we used ad-hoc methods to find our specimens, so no inference should be made from an entry not appearing.

\begin{tabular}{|c|c|c|c|}
\hline$n(\bmod 4)$ & $k=3$ & $k=4$ & $k=5$ \\
\hline 0 & 8 & 8 & 16 \\
1 & 5,9 & 9 & 9 \\
2 & 6 & $10,14,18$ & \\
3 & 3,7 & 7 & $11,19,23, \ldots$ \\
\hline
\end{tabular}

Table 3: Values of $(k, n)$ for which all plausible $\tau$-parities are achieved.

As Table 3 shows, we constructed explicit examples which achieve all plausible $\tau$-parities for $k \in\{3,4,5\}$ and each congruence class of $n \bmod 4$ (except for $n \equiv 2 \bmod 4$ when $k=5$ ). This exception is not a genuine one, since it will follow from Corollary 4.8 below that all plausible $\tau$-parities are actual for all sufficiently large $n$ when $k=5$.

Note that $n=9$ is the smallest order which achieves all plausible $\tau$-parities for $k=5$. Among the $\mathrm{OA}(6,9)$, there are 13312 actual $\tau$-parities, and 16384 plausible $\tau$-parities. The entry for $n \equiv 3(\bmod 4)$ and $k=5$ in Table 3 is justified by our next result. In it we construct an infinite family of orthogonal arrays which achieve all plausible $\tau$-parities when $k=5$ (and hence also for smaller $k$ ).

Theorem 4.4. For prime $n \geqslant 11$ satisfying $n \equiv 3(\bmod 4)$ there exist examples of $\mathrm{OA}(5, n)$ which achieve all 512 plausible $\tau$-parities.

Proof. Suppose $n \geqslant 11$ is prime and $n \equiv 3(\bmod 4)$. Let $A$ be the $\mathrm{OA}(n+1, n)$ corresponding to $n-1 \operatorname{MOLS}(n),\left\{L_{1}, \ldots, L_{n-1}\right\}$, where $L_{\lambda}[r, c]=\lambda r+c(\bmod n)$ for $\lambda \in[1, n-1]$. First we determine $\tau_{i j}^{c}$ for every $c, i, j \in[1, \ldots, k]$.

It is straightforward to check that $\tau_{i j}^{1}=0$ for every $i, j \in[2, k]$ since each of the permutations $\rho_{a}(1, i, j)$ is of the form $x \mapsto x+d(\bmod n)$ for a constant $d$. Observe that the parity of a permutation $x \mapsto b x+d$ is the same as the parity of $x \mapsto b x$. If $b$ has (multiplicative) order $q$ in $\mathbb{Z}_{n}$ then the permutation $x \mapsto b x$ has cycle structure consisting of $(n-1) / q$ cycles of length 
$q$. Hence it has even parity if and only if $(n-1)(q-1) / q$ is even, which occurs if and only if $q$ is odd, given that $n \equiv 3(\bmod 4)$. Now, $\rho_{a}(c, 1, j)$ is the permutation $x \mapsto(j-c) x+a$ for $j \geqslant 2$. For $j>i \geqslant 2$, we see that $\rho_{a}(c, i, j)$ is the permutation $(i-c) x+a \mapsto(j-c) x+a$, which has the same parity as the permutation $x \mapsto(i-c)^{-1}(j-c) x$. It follows that in $\mathbb{Z}_{2}$,

$$
\begin{aligned}
& \tau_{i j}^{1}=0, \\
& \tau_{1 j}^{c}=q-1, \text { for } j \geqslant 2, \text { where } q \text { is the order of } j-c, \text { and } \\
& \tau_{i j}^{c}=q-1, \text { for } j>i \geqslant 2, \text { where } q \text { is the order of }(i-c)^{-1}(j-c)
\end{aligned}
$$

Here order means multiplicative order, modulo $n$.

Let $a-1, a, a+1 \in \mathbb{Z}_{n}$ all be quadratic non-residues and define $A_{1}=\mathscr{A}\left(L_{a}, L_{a^{2}}, L_{a^{3}}\right)$. Thus, columns $1,2,3,4,5$ of $A_{1}$ correspond to columns $1,2, a+2, a^{2}+2, a^{3}+2$ of $A$. The entire vector $\tau\left(A_{1}\right)$ can be reconstructed once we work out that

$$
\left[\tau_{23}^{1}, \tau_{24}^{1}, \tau_{25}^{1}, \tau_{12}^{3}, \tau_{12}^{4}, \tau_{13}^{4}, \tau_{12}^{5}, \tau_{13}^{5}, \tau_{14}^{5}\right]=[0,0,0,0,1,1,0,0,0] .
$$

These values are not hard to derive. For example, $\tau_{13}^{5}$ for $A_{1}$ corresponds to $\tau_{1 j}^{c}$ for $A$ where $j=a+2$ and $c=a^{3}+2$. Since $j-c=a-a^{3}=(-a)(a+1)(a-1)$ is a quadratic residue, it follows that $q$ is odd and hence $\tau_{13}^{5}=0$ for $A_{1}$.

Let $a-1, a, a+1 \in \mathbb{Z}_{n}$ be such that $a$ is a quadratic non-residue and $a-1$ and $a+1$ are quadratic residues. Let $A_{2}=\mathscr{A}\left(L_{a}, L_{a^{2}}, L_{a^{3}}\right)$, so columns $1,2,3,4,5$ of $A_{1}$ correspond to columns $1,2, a+2, a^{2}+2, a^{3}+2$ of $A$. As before, we determine $\tau\left(A_{2}\right)$ by finding

$$
\left[\tau_{23}^{1}, \tau_{24}^{1}, \tau_{25}^{1}, \tau_{12}^{3}, \tau_{12}^{4}, \tau_{13}^{4}, \tau_{12}^{5}, \tau_{13}^{5}, \tau_{14}^{5}\right]=[0,0,0,0,1,0,0,0,1]
$$

Consulting our computation of the switching classes we find $\tau\left(A_{1}\right)$ in the switching class of size 192, while $\tau\left(A_{2}\right)$ belongs to the switching class of size 320 .

Jacobsthal $[12$ showed that for prime $n \equiv 3(\bmod 4)$, there exists a sequence of three consecutive elements which are quadratic non-residues for prime $n>7$ and there exists a sequence of consecutive elements which are residue, non-residue, residue for prime $n>3$. Thus, for prime $n \equiv 3(\bmod 4), n \geqslant 11$, there are elements $a-1, a, a+1 \in \mathbb{Z}_{n}$ such that the above constructions produce actual $\tau$-parities belonging to each of the two switching classes.

In principle, similar families can be constructed for other cases. However, examination of Table 1 reveals that the case that we solved in Theorem 4.4 involves fewer switching classes (and hence less work) than would be required for different values of $n(\bmod 4)$ or for larger values of $k$.

In the remainder of this section we demonstrate a different way to achieve infinite families that achieve all plausible $\tau$-parities. Specifically, we will show that if there are examples of $\mathrm{OA}(k, n)$ achieving all plausible $\tau$-parities for some $n$, then there are examples of $\mathrm{OA}(k, N)$ achieving all plausible $\tau$-parities for all sufficiently large $N$. We first require some definitions.

An incomplete Latin square $L=\left(l_{i j}\right)$ of order $n$ with a hole of order $h$ is an $n \times n$ array on an $n$-set $\Lambda$ with a hole $H \subseteq \Lambda$ such that each cell $(i, j)$ is empty if $\{i, j\} \subseteq H$ and contains exactly one symbol otherwise, every row and every column of $L$ contains each symbol at most once, and rows and columns indexed by $H$ do not contain symbols in $H$. A pair of incomplete Latin squares of order $n$ with common hole of order $h$ are orthogonal if, when superimposed, all of the $n^{2}-h^{2}$ ordered pairs of symbols in $\Lambda^{2} \backslash H^{2}$ occur amongst the cells $(i, j)$ where $\{i, j\} \nsubseteq H$. A set of incomplete Latin squares of order $n$ with a common hole of order $h$ which are pairwise orthogonal is called a set of incomplete MOLS.

An incomplete pairwise balanced design on $n$ points with hole size $h$ and blocks sizes $K$, denoted $I P B D((n, h), K)$, is a triple $(V, H, \mathscr{B})$ such that $V$ is a set of $n$ points, $H$ is a subset of $V$ of size $h$ called the hole, and $\mathscr{B}$ is a collection of subsets of $V$ where $|B| \in K$ for each 
$B \in \mathscr{B}$ and every pair of points not both in $H$ occur together in exactly one block. Combining a result from [5] (given as Construction 6.1.2 in [20]) and [20, Prop. 6.1.1], we have the following theorem.

Theorem 4.5. If there exists an $\operatorname{IPBD}((n, h), K)$ and for each $k \in K$ there exists $t+1 M O L S$ of order $k$, then there exist $t$ incomplete MOLS of order $n$ with a common hole of order $h$.

The following proof is due to Peter Dukes.

Theorem 4.6. For any fixed $t$ and $h$ and for all sufficiently large $n$, there exist $t$ incomplete $M O L S$ of order $n$ with a common hole of order $h$.

Proof. Let $K=\left\{2^{a}, 2^{a+1}, 3^{b}\right\}$ where $2^{a}>t+1$ and $3^{b}>t+1$. Then, by McNeish's Theorem (see, e.g. [20, Thm 1.1.2]), there exist $t+1$ MOLS of order $v$ for $v \in\left\{2^{a}, 2^{a+1}, 3^{b}\right\}$. Also, by [20, Thm 5.1.2] and the choice of $K$, there exists an $\operatorname{IPB} D((n, h), K)$ for all sufficiently large $n$. The result follows by Theorem 4.5,

We remark that Dukes and van Bommel [8] proved that there exists a set of $t$ incomplete MOLS of order $n$ with a common hole of order $h$ for all sufficiently large $n$ and $h$ satisfying $n \geqslant 8(t+1)^{2} h$.

Theorem 4.7. Let $N$ be sufficiently large relative to $n$. The number of actual $\tau$-parities for $(k, N)$ is no less than the number of actual $\tau$-parities for $(k, n)$.

Proof. Let $A_{1}$ and $A_{2}$ be two orthogonal arrays $\mathrm{OA}(k, n)$ with different $\tau$-parities. By Theorem 4.6, for all sufficiently large $N$, there exists an $\mathrm{OA}(k, N)$, say $A_{1}^{\prime}$, that contains $A_{1}$ as a subarray. Let $A_{2}^{\prime}$ be the $\mathrm{OA}(k, N)$ obtained from $A_{1}^{\prime}$ by replacing the subarray $A_{1}$ by $A_{2}$. Since $A_{1}$ and $A_{2}$ have different $\tau$-parities, there exist distinct $c, i, j \in[1, k]$ such that $\tau_{i j}^{c}\left(A_{1}\right) \neq \tau_{i j}^{c}\left(A_{2}\right)$. This forces $\tau_{i j}^{c}\left(A_{1}^{\prime}\right) \neq \tau_{i j}^{c}\left(A_{2}^{\prime}\right)$, so $A_{1}^{\prime}$ and $A_{2}^{\prime}$ have different $\tau$-parities. The result follows.

Corollary 4.8. If all plausible $\tau$-parities are actual for $(k, n)$ then, for all sufficiently large $N$, all plausible $\tau$-parities are actual for $(k, N)$.

On the basis of the evidence gathered above, we propose:

Conjecture 4.9. For any fixed $k$ and all sufficiently large $N$, all plausible $\tau$-parities are actual for $(k, N)$.

When $k$ and $n$ are comparable in size we might expect there to be plausible $\tau$-parities that are not actual for $(k, n)$. Indeed, we will see in the next section that this is definitely the case when $k=n+1$.

We close the section with an observation related to the comments at the end of \$2.1. If $M=\mathscr{M}(A)$ where $A$ is an $\mathrm{OA}(k, n)$, then the individual Latin squares in $M$ have at most $2(k-2)$ independent bits of information in their row, column and symbol parities. For $k \geqslant 4$, this is strictly less than $\left(\begin{array}{l}k \\ 2\end{array}\right)-1$, which suggests, on the basis of everything we have seen in this section, that a set of MOLS has more information in its $\tau$-parity than there is in the $\tau$-parity of its constituent Latin squares. Certainly, this is true for pairs and triples of MOLS of large orders, by combining Corollary 4.8 and Table 3 .

\section{$5 \quad$ Projective planes}

In this section, we study the important special case of orthogonal arrays which correspond to finite projective planes. To begin, we use the well-known fact that such orthogonal arrays correspond to sharply 2-transitive sets of permutations to derive a constraint on the $\tau$-parity 
of the OA. A set $S$ of permutations of a set $X$ is said to be sharply 2-transitive if for all $x, x^{\prime}, y, y^{\prime} \in X$ where $x \neq x^{\prime}$ and $y \neq y^{\prime}$, there exists a unique $\gamma \in S$ such that $\gamma(x)=y$ and $\gamma\left(x^{\prime}\right)=y^{\prime}$.

Theorem 5.1. Let $A=\left(a_{r \ell}\right)$ be an orthogonal array $\mathrm{OA}(n+1, n)$ on alphabet $\Lambda$, where $r \in \Lambda^{2}$ and $\ell \in[1, n+1]$. Let $i, j \in[1, n+1]$ be distinct integers. Then, in $\mathbb{Z}_{2}$,

$$
\sum_{c \in[1, n+1] \backslash\{i, j\}} \tau_{i j}^{c}=\left(\begin{array}{l}
n \\
2
\end{array}\right) .
$$

Proof. Let $C_{i j}=[1, n+1] \backslash\{i, j\}$. By definition, $\tau_{i j}^{c}=\sum_{x \in \Lambda} \pi\left(\rho_{x}(c, i, j)\right)$ for $c \in C_{i j}$. We exploit the fact that $\left\{\rho_{x}(c, i, j): x \in \Lambda, c \in C_{i j}\right\}$ is a sharply 2-transitive set of permutations. Indeed, let $u, u^{\prime}, v, v^{\prime} \in \Lambda$ be such that $u<u^{\prime}$ and $v>v^{\prime}$. Let $r, r^{\prime} \in \Lambda^{2}$ be such that $a_{r i}=u$, $a_{r j}=v, a_{r^{\prime} i}=u^{\prime}, a_{r^{\prime} j}=v^{\prime}$. No two rows of $A$ agree in more than one column, and overall there are $(n+1) n\left(\begin{array}{l}n \\ 2\end{array}\right)$ places in $A$ where two rows agree. Since this matches the number of pairs of rows, any two rows must agree in exactly one column. Thus, there is a unique $c \in C_{i j}$ such that $a_{r c}=a_{r^{\prime} c}=x$, and $\rho_{x}(c, i, j)$ maps $u \mapsto v$ and $u^{\prime} \mapsto v^{\prime}$, producing an inversion. So in $\mathbb{Z}_{2}$,

$$
\sum_{c \in C_{i j}} \tau_{i j}^{c}=\sum_{c \in C_{i j}} \sum_{x \in \Lambda} \pi\left(\rho_{x}(c, i, j)\right)=\left(\begin{array}{l}
n \\
2
\end{array}\right)^{2}=\left(\begin{array}{l}
n \\
2
\end{array}\right),
$$

because each of the possible $\left(\begin{array}{l}n \\ 2\end{array}\right)^{2}$ inversions occurs exactly once amongst the permutations in our sharply 2-transitive set.

By (5.1), we have a strengthening of Theorem 3.4 for the case when $k=n+1$.

Corollary 5.2. Let $G$ be the stack corresponding to an $\mathrm{OA}(n+1, n)$. Then $G$ is an empty graph when $n \equiv 0,1(\bmod 4)$ and $G$ is a complete graph when $n \equiv 2,3(\bmod 4)$.

Next we study the consequences of equation (5.1) on $\tau$ - and $\sigma$-graphs. First, we give an interpretation of this equation for a $\sigma$-graph. Then we use this fact to derive a property of the $\tau$-graphs.

Lemma 5.3. Let $A$ be an $\mathrm{OA}(n+1, n)$ with $\sigma$-graph $\mathscr{G}$.

(i) If $n \equiv 0(\bmod 4)$, then every vertex of $\mathscr{G}$ has even degree.

(ii) If $n \equiv 1(\bmod 4)$, then the degrees of the vertices of $\mathscr{G}$ all have the same parity.

(iii) If $n \equiv 2(\bmod 4)$, then every vertex of $G$ has odd in-degree and odd out-degree.

(iv) If $n \equiv 3(\bmod 4)$, then the in-degrees of the vertices of $\mathscr{G}$ are all of one parity and the out-degrees of the vertices of $\mathscr{G}$ are all of the other parity.

Proof. By the handshake lemma, it suffices to show that the (in-)degrees of the $n+1$ vertices in $\mathscr{G}$ all have the same parity. The parity of the (in-)degree of vertex $i \in[1, n+1]$ is $\pi\left(\prod_{c \neq i} \sigma_{c i}\right)$. Let $i, j \in[1, n+1]$ be two distinct vertices. Then, in $\mathbb{Z}_{2}$,

$$
\begin{aligned}
\pi\left(\prod_{c \neq i} \sigma_{c i}\right)+\pi\left(\prod_{c \neq j} \sigma_{c j}\right) & =\pi\left(\sigma_{i j} \sigma_{j i} \prod_{c \neq i, j} \sigma_{c i} \sigma_{c j}\right) & \\
& =\pi\left(\sigma_{i j} \sigma_{j i}\right)+\sum_{c \neq i, j} \tau_{i j}^{c} & \text { by (2.4) }
\end{aligned}
$$$$
=\left(\begin{array}{l}
n \\
2
\end{array}\right)+\left(\begin{array}{l}
n \\
2
\end{array}\right)=0 \quad \text { by (2.3) and (5.1) }
$$

Hence, the sum of the (in-)degrees of vertices $i$ and $j$ is even. Therefore, any two vertices $i$ and $j$ have the same parity of their (in-)degrees. 
Recall that every $\tau$-graph for an OA is a disjoint union of an isolated vertex and a complete bipartite graph. When we have an $\mathrm{OA}(n+1, n)$, the complete bipartite subgraphs in its $\tau$ graphs are induced on $n$ vertices. When $n$ is odd, one of the partite sets has an even number of vertices and the other has an odd number of vertices. The next theorem considers what happens when $n$ is even.

Lemma 5.4. Let $n$ be a positive even integer and suppose that a $\tau$-graph of an $\mathrm{OA}(n+1, n)$ is the disjoint union of an isolated vertex and $K_{n_{1}, n_{2}}$ for some nonnegative integers $n_{1}$ and $n_{2}$. Then $n_{1} \equiv n_{2} \equiv n / 2(\bmod 2)$.

Proof. We have that $n_{1}+n_{2}=n \equiv 0(\bmod 2)$ and the number of edges is $n_{1} n_{2}$. Hence, if there is an odd number of edges, then $n_{1} \equiv n_{2} \equiv 1(\bmod 2)$. Otherwise, $n_{1} \equiv n_{2} \equiv 0(\bmod 2)$. Let $c \in[1, n+1]$. Working in $\mathbb{Z}_{2}$, the parity of the number of edges in the $\tau$-graph $G_{c}$ is

$$
\sum_{i<j} \tau_{i j}^{c}=\pi\left(\prod_{i<j} \sigma_{c i} \sigma_{c j}\right)=(n-1) \pi\left(\prod_{i \neq c} \sigma_{c i}\right)= \begin{cases}0 & \text { if } n \equiv 0(\bmod 4), \\ 1 & \text { if } n \equiv 2(\bmod 4),\end{cases}
$$

by (2.4) and Lemma [5.3, where $i, j \in[1, n+1] \backslash\{c\}$.

When $k=n+1$, we call a plausible $\tau$-parity that satisfies (5.1) a PP-plausible $\tau$-parity. We next show that only some of the plausible $\tau$-parities are PP-plausible.

Theorem 5.5. The number of PP-plausible $\tau$-parities is $2^{\left(\begin{array}{l}n \\ 2\end{array}\right)}$ if $n$ is odd and $2^{\left(\begin{array}{l}n \\ 2\end{array}\right)-1}$ if $n$ is even.

Proof. We choose to use (2.5) for standardising $\sigma$-parity.

First suppose that $n$ is even. We can choose $\pi\left(\sigma_{i j}\right)$ for $1 \leqslant i<j \leqslant n$, except $\pi\left(\sigma_{12}\right)$. The values of $\pi\left(\sigma_{i j}\right)$ for $1 \leqslant j<i \leqslant n$ are then determined by (2.3). The values of $\pi\left(\sigma_{i j}\right)$ when $n+1 \in\{i, j\}$ can now be determined from Lemma 5.3. The $\sigma$-parity (and hence the $\tau$-parity) is thus determined by $\left(\begin{array}{l}n \\ 2\end{array}\right)-1$ binary choices. Next we argue that each of these options for the $\tau$-parity satisfies (5.1) and hence is PP-plausible. Fix $i, j \in[1, n+1]$ and let $\delta_{i}, \delta_{j}$ be, respectively, the (in-)degrees of vertices $i, j$ in the $\sigma$-graph. By construction, $\delta_{i}$ and $\delta_{j}$ agree $\bmod 2$. So, by (2.4) and (2.3), we have

$$
\sum_{c \in[1, n+1] \backslash\{i, j\}} \tau_{i j}^{c}=\sum_{c \in[1, n+1] \backslash\{i, j\}} \pi\left(\sigma_{c i} \sigma_{c j}\right)=\delta_{i}+\delta_{j}-\pi\left(\sigma_{i j}\right)-\pi\left(\sigma_{j i}\right)=\left(\begin{array}{l}
n \\
2
\end{array}\right)
$$

in $\mathbb{Z}_{2}$, confirming (5.1).

The situation for odd $n$ is similar except that we also get to choose $\pi\left(\sigma_{1(n+1)}\right)$. Once we have chosen $\pi\left(\sigma_{1 j}\right)$ for $1<j \leqslant n+1$ we know the parity of the (out-)degree of every vertex in the $\sigma$-graph, and we can proceed as for the even $n$ case.

Corollary 5.6. We have $\mathcal{B}(n+1, n) \leqslant\left(\begin{array}{l}n \\ 2\end{array}\right)$ if $n$ is odd and $\mathcal{B}(n+1, n) \leqslant\left(\begin{array}{l}n \\ 2\end{array}\right)-1$ if $n$ is even.

Comparing to Theorem 4.2, this means that not all plausible $\tau$-parities are actual $\tau$-parities for $(k, n)=(n+1, n)$. We have no way of judging how many PP-plausible $\tau$-parities are actual, although it is an interesting question.

\section{$6 \quad$ Parity of Latin squares in the ensemble}

In this section we primarily consider the parity of Latin squares in the ensemble of an orthogonal array. We will find various bounds and congruences that must be satisfied by the number of Latin squares of a particular parity in the ensemble. The results will depend on the congruence class of $n$ modulo 4 . 
While most of the section deals with properties of the ensemble, we begin with a result that constrains the number of each parity-type allowed among the $n-1$ Latin squares in a complete set of MOLS.

Theorem 6.1. Suppose there exist $n-1$ MOLS of order $n$ and let $z, y_{1}, y_{2}$ and $y_{3}$ be the number of these Latin squares of parity types 000, 011, 101 and 110, respectively, if $n \equiv 0,1(\bmod 4)$, or of parity types $111,100,010$ and 001 , respectively, if $n \equiv 2,3(\bmod 4)$. Then

(i) $z+y_{1}+y_{2}+y_{3}=n-1$, and

(ii) if $n$ is even then $y_{1} \equiv y_{2} \equiv y_{3} \not \equiv z(\bmod 2)$;

if $n \equiv 1(\bmod 4)$ then $y_{1} \equiv y_{2}$ and $y_{3} \equiv z(\bmod 2)$;

if $n \equiv 3(\bmod 4)$ then $y_{1} \not \equiv y_{2}$ and $y_{3} \not \equiv z(\bmod 2)$.

Moreover, if $z, y_{1}, y_{2}, y_{3} \in[0, n-1]$ satisfy (i) and (ii) then there is a PP-plausible $\tau$-parity corresponding to $n-1$ MOLS of order $n$ which include $z, y_{1}, y_{2}$ and $y_{3}$ Latin squares of the appropriate parity types.

Proof. We choose to use (2.5) for standardising $\sigma$-parity.

Let $M=\left\{M_{1}, \ldots, M_{n-1}\right\}$ be a set of MOLS of order $n$ and let $A=\mathscr{A}(M)$, which is an $\mathrm{OA}(n+1, n)$. Let $z, y_{1}, y_{2}$ and $y_{3}$ be the number of squares in $M$ that are of parity types 111 , 100, 010 and 001 , respectively if $n \equiv 2,3(\bmod 4)$, or of parity types $000,011,101$ and 110 , respectively if $n \equiv 0,1(\bmod 4)$. Clearly $(i)$ is satisfied.

By (2.3) and (2.4), for $c \in[3, n+1]$, we have $\tau_{2 c}^{1}=\pi\left(\sigma_{1 c}\right)$ and

$$
\tau_{1 c}^{2}= \begin{cases}1+\pi\left(\sigma_{2 c}\right) & \text { if } n \equiv 2,3(\bmod 4), \\ \pi\left(\sigma_{2 c}\right) & \text { if } n \equiv 0,1(\bmod 4)\end{cases}
$$

In the $\sigma$-matrix of $A$, the entries in the first two rows in column $c$ determine the parity type of $M_{c-2}$ for $c \in[3, n+1]$. These entries will be, respectively,

$(0,0),(0,1),(1,0)$ or $(1,1)$ if $M_{c-2}$ has parity type $000,011,101$ or 110 , or

$(1,0),(1,1),(0,0)$ or $(0,1)$ if $M_{c-2}$ has parity type $111,100,010$ or 001.

Thus, if $n \equiv 0,1(\bmod 4)$ the number of ones in the first row of the $\sigma$-matrix is $y_{2}+y_{3}$ and the number of ones in the second row is $y_{1}+y_{3}$. If $n \equiv 2,3(\bmod 4)$ the number of ones in the first row of the $\sigma$-matrix is $z+y_{1}$ and the number of ones in the second row is $1+y_{1}+y_{3}$, since $\pi\left(\sigma_{21}\right)=1$. Now (ii) follows from Lemma 5.3.

On the other hand, suppose $z, y_{1}, y_{2}, y_{3} \in[0, n-1]$ satisfy (i) and (ii). Define a matrix $W=\left(w_{i j}\right)$ or order $n+1$ such that $w_{12}=0$, and among the pairs $\left\{\left(w_{1 c}, w_{2 c}\right): c \in[3, n+1]\right\}$ there are, respectively, $z, y_{1}, y_{2}$ and $y_{3}$ occurrences of $(1,0),(1,1),(0,0)$ and $(0,1)$, if $n \equiv 2,3(\bmod 4)$ or $(0,0),(0,1),(1,0)$ and $(1,1)$, if $n \equiv 0,1(\bmod 4)$. As in the proof of Theorem 5.5 the remaining entries of $W$ above the main diagonal, except the last column, can be chosen to be either 0 or 1 ; then the last column and the remaining entries below the main diagonal can be determined such that $W$ is a $\sigma$-matrix corresponding to a PP-plausible $\tau$-parity. The condition (ii) ensures that the first two rows of $W$ have the same total, mod 2.

Corollary 6.2. There exists a PP-plausible $\tau$-parity corresponding to a complete set of equiparity MOLS if and only if $n \not \equiv 3(\bmod 4)$.

We stress that Corollary 6.2 only claims that for the corresponding $\mathrm{OA}(n+1, n)$, choosing the first two columns and any other column will create a subarray $B$ for which $\mathscr{M}(B)$ is equiparity. However, there will be Latin squares in the ensemble (arising from other choices of 
3 columns) which are not equiparity. For the rest of this section, we consider the number of equiparity Latin squares in the ensemble of an $\mathrm{OA}(k, n)$.

Next, we derive some equations which will be used in several of our proofs. Let $A$ be an $\mathrm{OA}(k, n)$ where $3 \leqslant k \leqslant n+1$ and $n \geqslant 2$. Let $T$ be the total number of edges among the $\tau$-graphs for $A$. Let $x$ denote the number of equiparity Latin squares in the ensemble of $A$, so $x$ is the number of 111 type Latin squares if $n \equiv 2,3(\bmod 4)$ or the number of 000 type Latin squares if $n \equiv 0,1(\bmod 4)$. We next relate $T$ and $x$. If $n \equiv 0,1(\bmod 4)$ then each non-equiparity Latin square in the ensemble contributes 2 to $T$, while equiparity Latin squares contribute nothing. If $n \equiv 2,3(\bmod 4)$ then each non-equiparity Latin square in the ensemble contributes 1 to $T$, while each equiparity Latin square contributes 3 . Thus,

$$
T= \begin{cases}2\left(\begin{array}{l}
k \\
3
\end{array}\right)-2 x & \text { if } n \equiv 0,1(\bmod 4) \\
2 x+\left(\begin{array}{l}
k \\
3
\end{array}\right) & \text { if } n \equiv 2,3(\bmod 4)\end{cases}
$$

We can also count $T$ using the row sums of the $\sigma$-matrix $M$, i.e. the adjacency matrix of the $\sigma$-graph. Let $\mu_{c}$ denote the sum of the entries in row $c$ of $M$. Observe that, by (2.4), $\tau_{i j}^{c}$ is 1 if and only if exactly one of $\pi\left(\sigma_{c i}\right)$ and $\pi\left(\sigma_{c j}\right)$ is 1 , for distinct integers $c, i, j \in[1, k]$. For a fixed integer $c$, the number of pairs of columns such that the cells in row $c$ (not including the main diagonal) have entries 0 and 1 , is the number of $\tau_{i j}^{c}$ which are 1 ; there are $\mu_{c}\left(k-1-\mu_{c}\right)$ such pairs. Thus,

$$
T=\sum_{c=1}^{k} \mu_{c}\left(k-1-\mu_{c}\right) \text {. }
$$

Next we consider each of the congruence classes for $n$ modulo 4 separately. When $n \equiv$ $0(\bmod 4)$, there are many examples of orthogonal arrays $\mathrm{OA}(n+1, n)$ for which every Latin square in the ensemble is an equiparity Latin square (necessarily of type 000). For example, as reported in [11], 18 of the 22 known projective planes of order 16 have the property that every associated $\mathrm{OA}(17,16)$ has only equiparity Latin squares in its ensemble. The only four that do not, namely MATH-D, JOHN-D, BBS4 and BBS4-D, still have the property that some associated $\mathrm{OA}(17,16)$ has only equiparity Latin squares in its ensemble. As an aside, we noticed that every one of the 22 projective planes has at least one associated complete set of MOLS in which each of the 15 Latin squares has all 48 permutations that contribute to the row, column and symbol parity being even. Such Latin squares are, of course, equiparity.

Clearly it is possible for all the Latin squares in an ensemble to be equiparity. However there are restrictions on the number of equiparity Latin squares in the ensemble.

Theorem 6.3. If $n \equiv 0(\bmod 4)$ then the number of equiparity Latin squares in the ensemble of an $\mathrm{OA}(n+1, n)$ is even and at least $n(n+1)(n-4) / 24$.

Proof. Suppose $n \equiv 0(\bmod 4)$ and let $A$ be an $\mathrm{OA}(n+1, n)$. Let $T$ be the total number of edges among the $\tau$-graphs of $A, \mu_{c}$ be the sum of the entries in row $c$ of the $\sigma$-matrix, and $x$ be the number of equiparity Latin squares in the ensemble of $A$. By (6.1) and (6.2),

$$
T=2\left(\begin{array}{c}
n+1 \\
3
\end{array}\right)-2 x=\sum_{c=1}^{n+1} \mu_{c}\left(n-\mu_{c}\right) \equiv 0(\bmod 4),
$$

where the congruence uses the fact that, by Lemma [5.3, $\mu_{c}$ is even for each $c \in[1, n+1]$. It follows that $x \equiv\left(\begin{array}{c}n+1 \\ 3\end{array}\right)(\bmod 2)$, and hence $x$ is even, since $n \equiv 0(\bmod 4)$. Finally, we note that $x$ is minimised when each $\mu_{c}=n / 2$ and $T=(n+1)(n / 2)^{2}$. So $x \geqslant n(n+1)(n-4) / 24$.

For $n \equiv 1(\bmod 4)$, the analogue of Theorem 6.3 is as follows, given that the maximum possible value of $T$ is $(n+1)^{2}(n-1) / 4$ : 
Theorem 6.4. If $n \equiv 1(\bmod 4)$ then the number of equiparity Latin squares in the ensemble of an $\mathrm{OA}(n+1, n)$ is at least $(n+1)(n-1)(n-3) / 24$.

In the case $n \equiv 1(\bmod 4)$, we cannot deduce whether the number of equiparity Latin squares is odd or even. Indeed, both possibilities occur amongst the PP-plausible $\tau$-parities. To see this, consider $\sigma$-graphs that are isomorphic to cycles of length 3 and 4 , respectively. By (6.1) and (6.2), the difference in the number of equiparity Latin squares between these two examples is $n-2$, which is odd.

Another difference between the cases $n \equiv 0(\bmod 4)$ and $n \equiv 1(\bmod 4)$ can be seen by considering a Desarguesian plane $\Pi$ of order $n$ if $n$ is a prime power. It is well-known that the collineation group of $\Pi$ is doubly-transitive. Given our comments in $\$ 1$, this means that every set of MOLS associated with $\Pi$ is isotopic. (Firstly, transitivity on lines ensures there is only one isomorphism class of OA associated with $\Pi$. Secondly, as a result of double transitivity, every ordered pair of columns of the OA is equivalent. The first two columns define the rows and columns of the Latin squares and the ordering of the other columns of the OA merely determines the ordering of the Latin squares, which is irrelevant in a set of MOLS.) The standard set of MOLS associated with $\Pi$ consists exclusively of Latin squares that are isotopic to the elementary abelian group. By the above comments, the same must be true of every set of MOLS derived from $\Pi$. If $\Pi$ has even order, this means that every Latin square in the ensemble must be equiparity since the Cayley table of any group is equiparity (see 91) and parity is an isotopism invariant for even orders, by Lemma 2.2. For odd orders the situation is different. In 44.1 , we saw that the Desarguesian plane of order 9 does not correspond to any OA $(10,9)$ for which the ensemble consists exclusively of equiparity Latin squares (although the other 3 projective planes of order 9 do!).

Unlike the case when $n \equiv 0,1(\bmod 4)$, it is impossible to build an $\mathrm{OA}(k, n)$ purely from equiparity Latin squares when $n \equiv 2,3(\bmod 4)$ and $k>3$.

Lemma 6.5. Suppose $n \equiv 2,3(\bmod 4)$ and $A$ is an $\mathrm{OA}(k, n)$. Let $B$ be any $\mathrm{OA}(4, n)$ formed from 4 columns of $A$. At most 2 of the 4 Latin squares in the ensemble of $B$ are equiparity.

Proof. Without loss of generality, consider columns $1,2,3,4$ and suppose the Latin squares defined by columns $1,2,3$ and by columns $1,2,4$ are of parity type 111 . Then $\tau_{34}^{1}=0=\tau_{34}^{2}$ by (2.2), so the other two squares are not of parity type 111.

This last result captures the intention behind Theorem 3.3 in [11. However, that Theorem was stated for Latin squares that are group-based. Such Latin squares are necessarily equiparity, as we noted in $\$ 1$. However, when $n \equiv 2(\bmod 4)$ they are well-known to not have any orthogonal mate, making Theorem 3.3 as stated in [11] vacuous. However, the class of equiparity Latin squares is far broader than the group-based Latin squares. Yet it turns out there cannot be too many of them in large OAs:

Theorem 6.6. The proportion of the Latin squares in the ensemble of an $\mathrm{OA}(k, n)$ that are equiparity is no more than $\frac{1}{4}+o(1)$ for $n, k \rightarrow \infty$ with $n \equiv 2,3(\bmod 4)$.

Proof. We can bound the number of equiparity Latin squares in an $\mathrm{OA}(k, n)$ using the fact that the $\tau$-graphs are all $K_{a, b}$ where $a+b=k-1$. The maximum number of edges in total amongst all $G_{c}$ is $k\left\lfloor\frac{k-1}{2}\right\rfloor\left\lceil\frac{k-1}{2}\right\rceil$. Thus, by (6.1), the number of equiparity Latin squares is at most

$$
\frac{1}{2}\left(k\lfloor(k-1) / 2\rfloor\lceil(k-1) / 2\rceil-\left(\begin{array}{l}
k \\
3
\end{array}\right)\right) .
$$

Hence asymptotically, the proportion of the $\left(\begin{array}{l}k \\ 3\end{array}\right)$ Latin squares that can be equiparity is at most $\frac{1}{4}+O(1 / k)$. 
We next show that for every $n \equiv 2,3(\bmod 4)$ the bound $(6.3)$ can be achieved by some PP-plausible $\tau$-parity.

Lemma 6.7. For each $n \equiv 2,3(\bmod 4)$, there exists a PP-plausible $\tau$-parity of an $\mathrm{OA}(n+1, n)$ such that each $\tau$-graph is isomorphic to $K_{1} \cup K_{\lfloor n / 2\rfloor,\lceil n / 2\rceil}$.

Proof. In this proof, for every integer $\xi$ we let $(\xi)_{n}$ denote the lowest non-negative integer that is congruent to $\xi \bmod n$. For distinct integers $i, j \in[1, n+1]$, define

$$
\pi\left(\sigma_{i j}\right)= \begin{cases}0 & \text { if }(j-i)_{n} \in[1,\lfloor n / 2\rfloor] \\ 1 & \text { otherwise. }\end{cases}
$$

By Theorem 4.2 and Lemma 5.3, this corresponds to a PP-plausible $\tau$-parity. In particular, by (2.4), for distinct integers $c, i, j \in[1, n+1]$,

$$
\tau_{i j}^{c}=\pi\left(\sigma_{c i} \sigma_{c j}\right)= \begin{cases}1 & \text { if precisely one of }(i-c)_{n} \text { and }(j-c)_{n} \text { is in }[1,\lfloor n / 2\rfloor] \\ 0 & \text { otherwise. }\end{cases}
$$

Thus, for each $c \in[1, n+1]$, the $\tau$-graph $G_{c}$ is the disjoint union of an isolated vertex and a complete bipartite graph with partite sets of size $\left\lfloor\frac{n}{2}\right\rfloor$ and $\left\lceil\frac{n}{2}\right\rceil$.

We have just considered the maximum value for the number of equiparity Latin squares in the ensemble of an $\mathrm{OA}(n+1, n)$. In the next two theorems, we derive a constraint in the form of a congruence and then a lower bound on this number. For the $n \equiv 3(\bmod 4)$ case in both results we invoke a form of standardisation different from (2.5). It will be more convenient for us to choose the parity of the out-degrees of the vertices in the $\sigma$-graph $\mathscr{G}$. By Lemma [3.6, $\mathscr{G}$ is a tournament and by Lemma 5.3. all out-degrees have the same parity. As $n+1$ is even, $\sigma$-complementation changes the parity of all out-degrees, so we can use it to select the parity that we want.

Theorem 6.8. If $n \equiv 2,3(\bmod 4)$ then the number of equiparity Latin squares in the ensemble of an $\mathrm{OA}(n+1, n)$ is congruent to $\lceil n / 4\rceil(\bmod 4)$.

Proof. Let $A$ be an $\mathrm{OA}(n+1, n)$. Let $x$ denote the number of equiparity Latin squares in the ensemble of $A$ and let $\mu_{c}$ denote the sum of the entries in row $c$ of the adjacency matrix of the $\sigma$-graph $\mathscr{G}$.

By Lemma 3.6, $\mathscr{G}$ is a tournament, and by Lemma [5.3, $\mu_{c}$ is odd for each $c \in[1, n+1]$ if $n \equiv 2(\bmod 4)$. If $n \equiv 3(\bmod 4)$, then by standardisation we may assume that the $\mu_{c}$ are all odd. Now, by (6.1) and (6.2),

$$
\begin{aligned}
2 x+\left(\begin{array}{c}
n+1 \\
3
\end{array}\right) & =\sum_{c=1}^{n+1} \mu_{c}\left(n-\mu_{c}\right) \\
& =n\left(\begin{array}{c}
n+1 \\
2
\end{array}\right)-\sum_{c=1}^{n+1} \mu_{c}^{2} \quad \text { as } \mathscr{G} \text { is a tournament } \\
& \equiv n\left(\begin{array}{c}
n+1 \\
2
\end{array}\right)-(n+1) \quad(\bmod 8),
\end{aligned}
$$

since each $\mu_{c}$ is odd. Hence, we find that

$$
x \equiv \begin{cases}\frac{(2 n-3)(n+1)}{3} \cdot \frac{n+2}{4} \equiv \frac{n+2}{4}(\bmod 4) & \text { if } n \equiv 2(\bmod 4), \\ \frac{(2 n-3)(n+2)}{3} \cdot \frac{n+1}{4} \equiv \frac{n+1}{4}(\bmod 4) & \text { if } n \equiv 3(\bmod 4) .\end{cases}
$$


We define a sequence $\mu_{1}, \ldots, \mu_{n+1}$ to be good if

$$
\sum_{i=1}^{m} \mu_{i} \leqslant n m-\left(\begin{array}{c}
m \\
2
\end{array}\right)
$$

for each $m \in[1, n+1]$. For our next theorem we will need the following property of good sequences:

Lemma 6.9. If $\mu_{1}, \ldots, \mu_{n+1}$ is a good sequence and $\mu_{c}=\mu_{c+1}-2$ for some $c \in[1, n]$ then the sequence obtained by interchanging $\mu_{c}$ with $\mu_{c+1}$ is also good.

Proof. Consider a hypothetical counterexample to the claim. Let $K=\sum_{i=1}^{c-1} \mu_{c}$. Applying (6.5) for $m \in\{c-1, c, c+1\}$, we see that

$$
\begin{gathered}
K \leqslant n(c-1)-\left(\begin{array}{c}
c-1 \\
2
\end{array}\right), \\
K+\mu_{c} \leqslant n c-\left(\begin{array}{l}
c \\
2
\end{array}\right)<K+\mu_{c}+2, \\
K+2 \mu_{c}+2 \leqslant n(c+1)-\left(\begin{array}{c}
c+1 \\
2
\end{array}\right) .
\end{gathered}
$$

It follows from (6.7) that $K+\mu_{c}=n c-\left(\begin{array}{l}c \\ 2\end{array}\right)-\varepsilon$ where $\varepsilon \in\{0,1\}$. Subtracting this equation from (6.8) gives $\mu_{c}+2 \leqslant n-c+\varepsilon$. Then, using (6.7) again, we get $K>n c-\left(\begin{array}{l}c \\ 2\end{array}\right)-n+c-\varepsilon=$ $n(c-1)-\left(\begin{array}{c}c-1 \\ 2\end{array}\right)+1-\varepsilon$, contradicting (6.6) $)$.

We can now derive a lower bound on the number of equiparity Latin squares in the ensemble.

Theorem 6.10. Let $n \equiv 2,3(\bmod 4)$. Any $\mathrm{OA}(n+1, n)$ has at least $\lceil n / 4\rceil$ equiparity Latin squares in its ensemble.

Proof. Let $A$ be an $\mathrm{OA}(n+1, n)$ and let $M$ be the $\sigma$-matrix of $A$. Let $\mu_{i}$ denote the total of the entries in row $i$ of $M$. In the $n \equiv 2(\bmod 4)$ case, each $\mu_{i}$ is odd by Lemma 5.3, In the $n \equiv 3(\bmod 4)$ case, by standardisation we can ensure that each $\mu_{i}$ is even. In either case, we have

$$
\mu_{i} \equiv n-1 \quad(\bmod 2)
$$

for each $i$. Let $T$ be the total number of edges among the $\tau$-graphs for $A$. The number of equiparity Latin squares in the ensemble of $A$ is $\left(T-\left(\begin{array}{c}n+1 \\ 3\end{array}\right)\right) / 2$ by (6.1), which will be minimised by minimising $T$. By (6.4), this is achieved by maximising $\sum \mu_{i}^{2}$. We may assume that $\mu_{1} \geqslant \mu_{2} \geqslant \cdots \geqslant \mu_{n+1}$ by relabelling if necessary. Also, (6.5) holds for each $m$ because the leading principal minor of order $m$ in $M$ has half its off-diagonal entries equal to zero.

Assume now that $\mu_{1}, \ldots, \mu_{n+1}$ is the monotonic good sequence that maximises $\sum \mu_{i}^{2}$ subject to (6.9). We claim that the sequence can be found with a greedy algorithm, in the sense that

$$
\sum_{i=1}^{m} \mu_{i} \geqslant n m-\left(\begin{array}{c}
m \\
2
\end{array}\right)-1
$$

for $1 \leqslant m \leqslant n+1$. Suppose not, and let $m=a$ be such that this inequality fails. Consider a new sequence $\mu_{1}^{\prime}, \ldots, \mu_{n+1}^{\prime}$ for which $\mu_{a}^{\prime}=\mu_{a}+2, \mu_{a+1}^{\prime}=\mu_{a+1}-2$, and $\mu_{i}^{\prime}=\mu_{i}$ for $i \notin\{a, a+1\}$. Clearly this new sequence is good, satisfies (6.9) and can be made monotonic by repeated application of Lemma 6.9. However,

$$
\sum_{i=1}^{m}\left(\mu_{i}^{\prime}\right)^{2}=\sum_{i=1}^{m} \mu_{i}^{2}+4\left(\mu_{a}-\mu_{a+1}\right)+8>\sum_{i=1}^{m} \mu_{i}^{2},
$$


contradicting the optimality of our original sequence. This proves our claim that the optimal sequence satisfies (6.10).

Note that there can be at most one monotonic good sequence satisfying $(6.9)$ and (6.10), because the constraints are such that each term of the sequence is determined by its predecessors. We next argue that this optimal sequence is given by $\mu_{1}=\mu_{2}=\mu_{3}=n-1, \mu_{4}=n-3$ and $\mu_{i}=\mu_{i-4}-4$ for $4<i \leqslant n+1$. Certainly this is a monotonic sequence of non-negative integers, since $\mu_{n+1}=n-1-4(n-2) / 4=1$ for $n \equiv 2(\bmod 4)$ and $\mu_{n+1}=n-3-4(n-3) / 4=0$ for $n \equiv 3(\bmod 4)$. Also, for $m=4 \alpha+\beta$ with $\beta \in\{1,2,3\}$, we have

$$
\begin{aligned}
\sum_{i=1}^{m} \mu_{i} & =4(n-1+n-5+\cdots+n-4 \alpha+3)-2 \alpha+\beta(n-4 \alpha-1) \\
& =4 \alpha(n-2 \alpha+1)-2 \alpha+\beta(n-1-4 \alpha)=n m-\left(\begin{array}{c}
m \\
2
\end{array}\right)+\left(\begin{array}{l}
\beta \\
2
\end{array}\right)-\beta .
\end{aligned}
$$

Since $\left(\begin{array}{l}\beta \\ 2\end{array}\right)-\beta \in\{-1,0\}$, we see that both (6.5) and (6.10) are satisfied in this case. These constraints are also satisfied in the case $m=4 \alpha$, because

$$
\sum_{i=1}^{m} \mu_{i}=4(n-1+n-5+\cdots+n-4 \alpha+3)-2 \alpha=4 \alpha(n-2 \alpha+1)-2 \alpha=n m-\left(\begin{array}{c}
m \\
2
\end{array}\right) .
$$

Returning to (6.4), we now know that

$$
\begin{aligned}
T & \geqslant n\left(\begin{array}{c}
n+1 \\
2
\end{array}\right)-3 \sum_{i=0}^{\lfloor(n-1) / 4\rfloor}(n-1-4 i)^{2}-\sum_{i=0}^{\lfloor(n-3) / 4\rfloor}(n-3-4 i)^{2} \\
& = \begin{cases}n^{3} / 6+n / 3+1 & \text { if } n \equiv 2(\bmod 4), \\
n^{3} / 6+n / 3+1 / 2 & \text { if } n \equiv 3(\bmod 4),\end{cases}
\end{aligned}
$$

which then implies the result, via (6.4) and (6.1).

Corollary 6.11. If $n \equiv 2(\bmod 4)$ and $n>2$ then the ensemble of an $\mathrm{OA}(n+1, n)$ contains at least two Latin squares that are not isotopic to each other.

Proof. By Theorem 6.10 the ensemble contains an equiparity Latin square. However, given that $n>2$, Lemma 6.5 tells us that the ensemble also contains a Latin square which is not equiparity. Parity is an isotopism invariant for even orders.

In our definition of ensemble we have insisted that each set of three columns produces a single Latin square. If we had instead allowed the three columns to be used in any order then 6 potentially different Latin squares would be produced for each set of three columns. Corollary 6.11 could then be strengthened to say that there will be at least 4 isotopism classes among the Latin squares corresponding to an $\mathrm{OA}(n+1, n)$ when $n \equiv 2(\bmod 4)$, since each of the parity types $001,010,100,111$ must occur.

It is also worth noting that there is a PP-plausible $\tau$-parity that achieves the bound in Theorem 6.10. We can build the adjacency matrix $M$ for the corresponding $\sigma$-graph as follows. Let

$$
B_{3}=\left[\begin{array}{lll}
0 & 0 & 1 \\
1 & 0 & 0 \\
0 & 1 & 0
\end{array}\right] \text { and } B_{4}=\left[\begin{array}{llll}
0 & 0 & 1 & 1 \\
1 & 0 & 0 & 1 \\
0 & 1 & 0 & 1 \\
0 & 0 & 0 & 0
\end{array}\right]
$$

If $n=4 \alpha+2$, place $\alpha$ copies of the block $B_{4}$ and one copy of $B_{3}$ down the diagonal of $M$; if $n=4 \alpha+3$, place $\alpha+1$ copies of $B_{4}$ down the diagonal of $M$. Fill all other entries above the 
diagonal with 1's and all those below with 0's. It is routine to check that this construction has the required properties, including achieving the optimal sequence $\left\{\mu_{i}\right\}$.

In contrast to Theorem 6.10, there are plausible $\tau$-parities for which the ensemble contains no equiparity Latin squares.

Lemma 6.12. If $n \equiv 2,3(\bmod 4)$ and $k$ is arbitrary, then there exists a plausible $\tau$-parity for which there are no equiparity Latin squares in the ensemble of the associated $\mathrm{OA}(k, n)$.

Proof. Take the $\sigma$-matrix to be lower triangular. Then the $\tau$-parities (for $i<j$ ) are given by:

$$
\tau_{i j}^{c}= \begin{cases}0 & \text { if } i<j<c \text { or } c<i<j \\ 1 & \text { if } i<c<j\end{cases}
$$

For any three distinct integers $c, i, j \in[1, k]$, exactly one of $\tau_{j c}^{i}, \tau_{i c}^{j}, \tau_{i j}^{c}$ is 1 , and the result follows.

We stress that the plausible $\tau$-parities in Lemma 6.12 are not PP-plausible, by Lemma 5.3 . Most, though not all, of the restrictions that we have demonstrated on the parities of Latin squares in the ensemble only apply to the projective plane case.

\section{Concluding remarks}

We have considered two notions of parity for the orthogonal arrays that correspond to MOLS. One of these, $\sigma$-parity, was introduced by Glynn and Byatt [11 in a more limited setting. The other, $\tau$-parity, is a direct generalisation of the row, column and symbol parities of Latin squares. These two notions of parity are closely related; $\tau$-parity is determined by $\sigma$-parity, and the converse is true up to complementation (see \$2.3). The relationship between the two parities proved very fruitful throughout our investigations. For example, it provided a very simple proof of Lemma 2.6, which generalises the well-known relationship between the row, column and symbol parities of Latin squares.

In 93 we introduced useful graph theoretic models for the two notions of parity. Each $\mathrm{OA}(k, n)$ has one $\sigma$-graph, $k \tau$-graphs and one "stack" which is formed by merging the $\tau$ graphs, modulo 2 . The $\sigma$-graph is undirected for $n \equiv 0,1(\bmod 4)$, but is a tournament for $n \equiv 2,3(\bmod 4)($ Lemma 3.6$)$. The $\tau$-graphs and stack are highly structured (Lemma 3.2, Theorem 3.4). Further restrictions apply to the structure of $\sigma$-graphs, $\tau$-graphs and the stack for OAs that come from projective planes (Lemma 5.3, Lemma 5.4, Corollary 5.2). These restrictions are based on the equivalence between projective planes and sharply 2-transitive sets of permutations.

In 84 we considered the question of how many different $\tau$-parities might be obtained by an $\mathrm{OA}(k, n)$. We phrased this in terms of $\mathcal{B}(k, n)$, the information content of the $\tau$-parity, in bits. We showed that $\mathcal{B}(k, n) \leqslant\left(\begin{array}{l}k \\ 2\end{array}\right)-1$ (Corollary 4.3). Later we showed that $\mathcal{B}(n+1, n) \leqslant\left(\begin{array}{l}n \\ 2\end{array}\right)$ if $n$ is odd and $\mathcal{B}(n+1, n) \leqslant\left(\begin{array}{l}n \\ 2\end{array}\right)-1$ if $n$ is even (Corollary 5.6).

An interesting question is whether there are restrictions on parities of an $\operatorname{OA}(k, n)$ other than the ones that we have demonstrated. This question is wide open for the case when $k$ is comparable in size to $n$. However, we conjecture that when $n$ is large relative to $k$, no further restrictions apply (Conjecture 4.9). We proved this conjecture for $k \leqslant 5$ in $\$ 4.2$ by showing that all plausible $\tau$-parities are actually achieved. Moreover, it is possible to embed any $k$-MOLS of order $n$ inside some set of $k$-MOLS of order $N$ whenever $N$ is large enough. Hence if $(k, n)$ is such that $\mathcal{B}(k, n)$ achieves its upper bound then $\mathcal{B}(k, N)=\mathcal{B}(k, n)$ for all large $N$.

An open question, related to the issue of whether there are further constraints when $k$ is comparable to $n$, is the issue of unique completion. It was shown by Metsch [18] that any 
$\mathrm{OA}\left(n-O\left(n^{1 / 3}\right), n\right)$ has a unique completion to an $\mathrm{OA}(n+1, n)$, up to isomorphism. It would be interesting to find parity analogues of this result. Examining the proof of Theorem [5.5, we see that the $\sigma$-parity of an $\mathrm{OA}(n, n)$ determines the $\sigma$-parity of its completion to an $\mathrm{OA}(n+1, n)$, but the same is not true for an $\mathrm{OA}(n-1, n)$ without further developments in the theory.

In $\$ 6$ we considered the ensemble of an $\mathrm{OA}(k, n)$, which is the set obtained by taking 3 columns of the $\mathrm{OA}$ at a time and interpreting the result as a Latin square. It turns out that there are many bounds and congruences that restrict the number of Latin squares of each parity that may occur within the ensemble. The restrictions, which mostly apply when $k=n+1$, are weakest for $n \equiv 0,1(\bmod 4)$. Many of the results in 66 that apply to $n \equiv 2(\bmod 4)$ also apply to $n \equiv 3(\bmod 4)$, but are not so limiting in that case since parity is not an isotopism invariant for odd $n$. Hence, our constraints are strictest when $n \equiv 2(\bmod 4)$, perhaps offering some insight into why projective planes of these orders are hard to construct (and are believed by many not to exist for $n>2$ ). In Corollary 6.11 we showed that all the Latin squares in the ensemble cannot be isotopic to each other when $n \equiv 2(\bmod 4)$. Indeed, as $n$ grows the number of "equiparity" Latin squares in the ensemble grows at least linearly (Theorem 6.8), but it can never be more than $\frac{1}{4}+o(1)$ of all the Latin squares in the ensemble (Theorem 6.6).

The preponderance of even parities among the known projective planes of order 16 invites further investigation. Certainly, for $n \equiv 0(\bmod 4)$ all of the constraints that we have found are trivially satisfied when all parities are even. This means the $\sigma$-graph, all $\tau$-graphs and the stack are all empty graphs, which seems to be the easiest way to satisfy all known requirements. In contrast, for $n \equiv 2(\bmod 4)$ all these graphs are forced to have edges, and the constraints such as Lemma 5.4 and Corollary 5.2 seem at first glance to be much harder to satisfy. However, it is worth stressing that in Theorem 5.5 we have a mechanism for producing many choices for the parities that satisfy all the constraints that we have demonstrated in this paper. Our work cannot be used to rule out the existence of any projective plane without the discovery of a new constraint on its parity.

\section{Acknowledgements}

The authors are very grateful to Peter Dukes for supplying Theorem 4.6 and to Darcy Best for helpful proofreading.

\section{References}

[1] R. Aharoni, M. Loebl, The odd case of Rota's bases conjecture, Adv. Math. 282 (2015), 427-442.

[2] L. Alpoge, Square-root cancellation for the signs of Latin squares, Combinatorica, to appear. DOI: $10.1007 / \mathrm{s} 00493-015-3373-7$.

[3] A. E. Brouwer, A. M. Cohen and A. Neumaier, Distance-Regular Graphs, Springer, Berlin, 1989.

[4] N. J. Cavenagh and I. M. Wanless, There are asymptotically the same number of Latin squares of each parity, Bull. Aust. Math. Soc. 94 (2016), 187-194.

[5] C. J. Colbourn and J.H. Dinitz, Mutually orthogonal Latin squares: A brief survey of constructions, J. Statist. Plann. Inference, 95 (2001), 9-48.

[6] C. J. Colbourn and J.H. Dinitz (eds), Handbook of combinatorial designs, 2nd ed., Chapman \& Hall/CRC, Boca Raton, FL, 2007. xxii+984 pp.

[7] D. M. Donovan, M. J. Grannell, T. S.Griggs and J. G. Lefevre, On parity vectors of Latin squares, Graphs Combin. 26 (2010) 673-684.

[8] P. J. Dukes and C. M. van Bommel, Mutually orthogonal Latin squares with large holes, J. Statist. Plann. Inference, 159 (2015), 81-89. 
[9] J. Egan and I. M. Wanless, Enumeration of MOLS of small order, Math. Comp. 85 (2016), 799824.

[10] D. G. Glynn, The conjectures of Alon-Tarsi and Rota in dimension prime minus one, SIAM J. Discrete Math. 24 (2010), 394-399.

[11] D. G. Glynn and D. Byatt, Graphs for orthogonal arrays and projective planes of even order, SIAM J. Discrete Math. 26 (2012), No. 3, 1076-1087.

[12] E. Jacobsthal, Anwendungen eiuer formel aus der theorie der quadratischen reste, Dissertation (Berlin, 1906), 26-32.

[13] J. C. M. Janssen, On even and odd Latin squares, J. Combin. Theory Ser. A, 69 (1995) 173-181.

[14] P. Kaski, A. D. S. Medeiros, P. R. J. Östergård and I. M. Wanless, Switching in one-factorisations of complete graphs, Electron. J. Comb. 21(2) (2014), \#P2.49.

[15] A. D. Keedwell and J. Dénes, Latin squares and their applications (2nd ed.), North Holland, Amsterdam, 2015.

[16] D. Kotlar, Parity types, cycle structures and autotopisms of Latin squares, Electron. J. Combin. 19(3) (2012), \#P10, 17 pp.

[17] B. D. McKay and P. Schweitzer, Switching reconstruction of digraphs, J. Graph Theory 76 (2014), 279-296.

[18] K. Metsch, Improvement of Bruck's completion theorem, Des. Codes Cryptogr. 1 (1991), 99-116.

[19] N. J. A. Sloane, The On-Line Encyclopedia of Integer Sequences, http://www.research.att.com/ njas/sequences/.

[20] C. M. van Bommel, An asymptotic existence theory on incomplete mutually orthogonal Latin squares, Master's thesis, Uni. Victoria, (2015).

[21] D.S. Stones and I. M. Wanless, How not to prove the Alon-Tarsi conjecture, Nagoya Math. J. 205 (2012), 1-24.

[22] I. M. Wanless, Cycle switches in Latin squares, Graphs Combin. 20 (2004) 545-570.

[23] I. M. Wanless, Data on Mutually Orthogonal Latin Squares (MOLS) http://users.monash.edu.au/ iwanless/data/MOLS/.

[24] P. Zappa, Triplets of Latin squares, Bollettino U.M.I. (7) 10-A (1996) 63-69. 